Article

\title{
Inter-Varietal Diversity of Typical Volatile and Phenolic Profiles of Croatian Extra Virgin Olive Oils as Revealed by GC-IT-MS and UPLC-DAD Analysis
}

\author{
Igor Lukić ${ }^{1,2, * \mathbb{C}}$, Marina Lukić ${ }^{1}$, Mirella Žanetić ${ }^{3}$, Marin Krapac ${ }^{1} \mathbb{D}$, Sara Godena ${ }^{1}(\mathbb{D}$ and \\ Karolina Brkić Bubola ${ }^{1}$ \\ 1 Institute of Agriculture and Tourism, Karla Huguesa 8, HR-52440 Poreč, Croatia; marina@iptpo.hr (M.L.); \\ marin@iptpo.hr (M.K.); sara@iptpo.hr (S.G.); karolina@iptpo.hr (K.B.B.) \\ 2 Centre of Excellence for Biodiversity and Molecular Plant Breeding, Svetošimunska 25, \\ HR-10000 Zagreb, Croatia \\ 3 Institute for Adriatic Crops and Karst Reclamation, Put Duilova 11, HR-21000 Split, Croatia; \\ mirella.zanetic@krs.hr \\ * Correspondence: igor@iptpo.hr; Tel.: +385-52-408-327
}

Received: 11 October 2019; Accepted: 6 November 2019; Published: 9 November 2019

\begin{abstract}
Despite having an interesting native olive gene pool and a rapidly emerging olive oil industry, monovarietal extra virgin olive oils (EVOO) from Croatia are relatively unexplored. To investigate the inter-varietal diversity of typical volatile and phenolic profiles of Croatian EVOO, 93 samples from six olive (Olea europaea L.) varieties were subjected to gas chromatography-ion trap mass spectrometry (GC-IT-MS) and ultra-performance liquid chromatography with diode array detection (UPLC-DAD), respectively. Quantitative descriptive sensory analysis was also performed. Analysis of variance extracted many relevant exclusive or partial discriminators between monovarietal EVOOs among the identified volatile compounds and phenols. Successful differentiation model with a $100 \%$ correct classification was built by linear discriminant analysis, while the most typical volatiles for each monovarietal EVOO were confirmed by partial least squares discriminant analysis. Diverse typical sensory attributes among the EVOOs were tentatively ascribed to the variations in the composition of volatiles and phenols. It was proven that the approach that comprises GC-IT-MS and UPLC-DAD analysis may provide additional objective information about varietal origin and typicity which successfully complement those obtained by sensory analysis. The approach was characterized as universal in nature, with a significant potential to contribute in strengthening the varietal identities and position on the market of monovarietal and Protected Denomination of Origin (PDO) EVOO.
\end{abstract}

Keywords: extra virgin olive oil; volatile compounds; phenols; sensory quality; varietal typicity

\section{Introduction}

Extra virgin olive oil (EVOO) is appreciated among consumers because of its specific flavor and nutritional properties. Due to its economic importance, EVOO is among the most common commodities subject to fraud and mislabeling. The European Union (EU) protects EVOO by the regulation mostly based on analytical and sensory controls [1] which generally succeed in detecting illegal manipulation with EVOO intrinsic properties (adulteration with cheaper refined and/or extraneous oils) and EVOO extrinsic properties (fraudulent misrepresentation of quality category). In the EU, most EVOOs of high economic value are additionally protected by Protected Denomination of Origin (PDO) [2]. Each PDO EVOO is produced according to a set of specific requirements prescribed by the holder of a designation in a specification document, governing aspects such as olive varieties used, cultivation, harvest and processing conditions, physico-chemical parameters, and sensory characteristics. 
Many PDO EVOOs are produced from olives of a single variety (monovarietal EVOOs), while the blends also owe a large part of their typicity to the unique olive assortment of a particular region. Nowadays, the information on the label about the varietal origin of EVOO is becoming more and more important and attracting, especially for the market segment of informed consumers interested in healthy, quality products with remarkable diversity and clear identity. Similar as in the case of wine, besides being linked to a given geographical origin and PDO, EVOOs from particular varieties are recognized, appreciated, and demanded on the market because of their specific nutritional and sensory properties. As a consequence, they often reach higher prices, and, given the obvious financial benefits associated with them, are very likely subject to fraud by mislabeling with respect to varietal origin.

As regards EVOO varietal authentication within the process of protection by designation of origin (PDO), controls against counterfeiting include auditing of mandatory documentation and records that prove traceability in production and compliance with the requirements set up in PDO specification. The other part is the assessment of the conformity of EVOO with physico-chemical parameters and sensory characteristics laid down in the specification. The analytical parameters controlled more often (e.g., acidity, peroxide value, measurements in ultraviolet, etc.) do not specifically reflect varietal origin, and the limits that are established, although usually stricter than those prescribed by the official EU regulation [1], are regularly not designed to identify olive variety used. Similar applies for the sensory profiles commonly used to describe monovarietal/PDO EVOO [3] which are not highly discriminative.

The mentioned measures are not sufficient to control varietal origin and avoid fraud. Fraud or false labelling might also be detected or confirmed chemically by analysis of other, minor EVOO compounds. The general strategy that is followed in various research laboratories is the detection of as many as possible EVOO constituents from a larger set of samples and application of multivariate statistical analysis to the analytical data in order to build up classification/prediction models based on varietal origin $[4,5]$. Many EVOO compounds were found useful for this purpose, including sterols [6], tocopherols [7], fatty acids [6,8], etc. The chemical compounds whose amounts are regulated neither by the official EU regulation nor the PDO specifications, but are certainly the most involved in the typical sensory identity of PDO and monovarietal EVOOs and could serve as differentiators based on such criteria, are volatile aroma compounds and phenols [9-14]. In fact, many successful reports were published which confirmed the utility of these constituents for EVOO varietal differentiation [12,14-20]. Volatile fraction of high quality EVOO, which is responsible for its characteristic so-called green and fruity flavor, consists mainly of $\mathrm{C} 5$ and $\mathrm{C} 6$ volatiles (aldehydes, ketones, alcohols, and esters) generated enzymatically in the so-called lipoxygenase (LOX) pathway and other subsequent bioprocesses during olive processing. LOX-derived compounds are accompanied by those from other chemical classes, such as hydrocarbons, terpenes, benzenoids, etc. with mostly unknown or minor sensory relevance [12,14,21-23]. Besides being among the most important contributors to EVOO antioxidant activity, phenols, especially secoiridoids which are the most abundant, are responsible for the characteristic EVOO bitterness and pungency [12-14,24,25]. Olive oil phenols are formed mainly by cleavage of their glycosides by hydrolytic enzymes during olive fruit processing and their concentrations are further affected by oxidative degradation catalyzed by polyphenoloxidases and peroxidases [26,27]. The activity of the mentioned enzymes responsible for the formation of both volatile compounds and phenols is strongly genetically predetermined [28,29], which makes these compounds a logical choice for potential varietal markers in EVOO varietal characterization and differentiation studies.

Croatia is the latest country that joined EU in 2013, and some of the most recently registered PDOs are Croatian [30]. Despite relatively small quantities produced in relation to the leading olive oil producing countries, such as Spain, Italy, Greece etc. [31], EVOOs from Croatia are emerging rapidly on the global market and are much appreciated. For example, Croatian EVOOs are often among those awarded with the highest prizes at relevant international competitions, while Istria, one of the most important olive growing and EVOO producing regions in Croatia, has been represented in the first and leading global EVOO guide Flos Olei by the largest number of EVOOs among all the regions for the last 
four years in a row (2015-2018). The olive plantations in Croatia have high genetic diversity, including many native varieties which concentrate close to their area of origin and show a limited geographical dispersion [32]. For this reason, Croatian EVOOs protected by various PDOs owe a significant part of their typicity to the varietal origin of the olives, which certainly becomes most pronounced in the case of monovarietal EVOO. In spite of that, and despite existing reports on the chemical and sensory characteristics of Croatian monovarietal EVOO [33-40], the potential of Croatian native olive varieties to produce diverse and specific EVOO has not been investigated enough to be adequately exploited in designing more unique and robust PDOs.

The main aim of this study was to investigate the inter-varietal diversity of typical volatile and phenolic profiles of Croatian monovarietal EVOOs by gas chromatography-ion trap mass spectrometry (GC-IT-MS) and ultra-performance liquid chromatography with diode array detection (UPLC-DAD), respectively. The approach was tested for the characterization and differentiation of EVOOs made from native varieties grown in the two most important olive growing regions in Croatia, Istria and Dalmatia, with each monovarietal EVOO represented by a heterogeneous sample group in terms of geographical microlocations, growing conditions, harvest date, olive processing technology, and EVOO finalization and storage parameters. It was expected that the results obtained would be useful for improving the understanding of the origins of the typical sensory characteristics of the investigated Croatian monovarietal EVOOs. However, the main premise was that the instrumental techniques utilized would be effective in tracing robust chemical markers among the investigated compounds despite the aforementioned sample heterogeneity, able to provide complementary information about varietal origin to that obtained by sensory analysis. Besides allowing better quality management and control in production, such findings would contribute strengthening the PDO identities and position on the market of Croatian EVOO.

\section{Materials and Methods}

\subsection{EVOO Samples}

For this study, the most economically important and widespread Croatian native olive varieties (Olea europaea L.) were considered. Representative monovarietal EVOO samples, made from Buža (19 samples), Istarska bjelica (22 samples), and Rosinjola (8 samples) olive varieties specific for the region of Istria, and Oblica (15 samples) and Lastovka (10 samples) olive varieties specific for the region of Dalmatia, were collected from local producers. In addition, representative monovarietal EVOO samples from a widespread variety Leccino (19 samples) grown in Istria were also collected. Detailed climatological data for Istrian and Dalmatia regions in year 2015 are reported in Table S1. EVOOs were selected to cover the maximum possible variability of each production area, and all the samples from the same variety were produced by different producers. Olive fruit samples were hand-picked at the usual maturity level for each cultivar during the local customary harvest period during October/November 2015. The collected EVOO samples were produced in various private mills using contemporary oil extraction equipment with temperature of malaxation kept below $27^{\circ} \mathrm{C}$. After finalization (clarification and storage), market-ready EVOOs were kept at low temperature in amber dark glass bottles prior to analysis, and analyzed during a period of 3 months.

\subsection{Sensory Analysis}

Quantitative descriptive analysis of EVOO samples was performed by the Panel for sensory analysis of VOO of the Institute of Agriculture and Tourism in Poreč (Croatia), accredited for VOO sensory analysis according to the EN ISO/IEC 17025:2007 standard and authorized by the Croatian Ministry of Agriculture for official VOO testing from 2012, and recognized in continuation by the IOC from 2014. The panel consisted of eight assessors (4 female, 4 male, average age 39) trained and accredited for VOO sensory analysis according to the International Olive Council (IOC) method adopted by the European Commission Regulation [1]. As well, all the tasters have had long-term 
involvement in EVOO research and have gained large experience in Croatian monovarietal EVOO sensory analysis. Qualitative (selection of descriptors/attributes by consensus and standardization of vocabulary) and quantitative (intensity of perception) criteria of the tasters were attuned by audibly tasting representative samples of Croatian monovarietal EVOOs through several preliminary training sessions. The panel agreed that the sensory attributes which best describe the investigated monovarietal EVOOs were the same for all varieties and among those commonly perceived in EVOO, but differed with respect to the ratios of their intensities. The panel used a modified profile sheet expanded with particular positive odor and taste attributes, which were quantified using a $10 \mathrm{~cm}$ unstructured intensity ordinal rating scale from 0 (no perception) to 10 (the highest intensity). For evaluating general quality attributes, a 10-point overall structured rating scale from 0 (the lowest quality) to 10 (the highest quality) was applied. For overall quality evaluation, VOOs were graded with points from 1 (the lowest quality) to 9 (the highest quality). Before each session, the tasters attuned their criteria with respect to the intensities of the perceived sensory attributes by tasting the same standard reference VOO sample, a blend characterized by all the selected sensory attributes/descriptors. According to the sensory analysis, all the investigated samples were classified as EVOO (no defect, fruitiness $>0$ ).

\subsection{Chemical Standards and Standard Solutions}

Methanol, water, and n-hexane were of HPLC grade purity (Sigma-Aldrich, St. Louis, MO, USA). Pure chemical standards of volatile compounds and phenols were purchased from AccuStandard Inc. (New Haven, CT, USA), Acros Organics (Geel, Belgium), Alfa Aesar (Haverhill, MA, USA), Cayman Chemical Co. (Ann Arbor, MI, USA), Extrasynthese (Genay, France), Fluka (Buchs, Switzerland), Honeywell International Inc. (Morris Plains, NJ, USA), Merck (Darmstadt, Germany), and Sigma-Aldrich. Standard solutions of volatiles were prepared in refined sunflower oil and that of phenols in pure methanol.

\subsection{Analysis of Volatile Compounds by GC-IT-MS}

Volatile compounds were isolated using headspace solid-phase microextraction (HS-SPME), according to the modified method proposed by Brkić Bubola, Koprivnjak, Sladonja, Škevin, and Belobrajić [41]. SPME fiber used was divinylbenzene/carboxen/polydimethylsiloxane (DVB/CAR/PDMS), $1 \mathrm{~cm}$ length, 50/30 $\mu \mathrm{m}$ film thickness (Supelco, Bellefonte, PA, USA). Four grams of EVOO sample (or a standard solution) were placed in a $10 \mathrm{~mL}$ glass vial containing a micro-stirring bar, and sealed. The headspace in the vial was equilibrated at $40{ }^{\circ} \mathrm{C}$ for $15 \mathrm{~min}$, and the extraction was carried out at $40^{\circ} \mathrm{C}$ for $40 \mathrm{~min}$ with stirring at $800 \mathrm{rpm}$. Thermal desorption of analytes was achieved in the GC injection port in splitless mode at $245^{\circ} \mathrm{C}$ for $3 \mathrm{~min}$. Identification and quantification of volatile compounds was performed using a Varian 3900 GC coupled to a Varian Saturn $2100 \mathrm{~T}$ ion trap mass spectrometer (IT-MS) (Varian Inc., Harbor City, CA, USA). A capillary column Rtx-WAX $(60 \mathrm{~m} \times 0.25 \mathrm{~mm}$ i.d. $\times 0.25 \mu \mathrm{m}$ film thickness; Restek, Bellefonte, PA, USA) was used. Initial oven temperature was $40{ }^{\circ} \mathrm{C}$, increased to $210{ }^{\circ} \mathrm{C}$ at $2{ }^{\circ} \mathrm{C} / \mathrm{min}$, increased to $245^{\circ} \mathrm{C}$ at $20^{\circ} \mathrm{C} / \mathrm{min}$, and kept for $20 \mathrm{~min}$. Injector, transfer line and ion trap temperatures were 245,180 , and $120^{\circ} \mathrm{C}$, respectively. Mass spectra were acquired in EI mode $(70 \mathrm{eV})$ at $1 \mathrm{~s} / \mathrm{scan}$, full scan with a range of $30-450 \mathrm{~m} / \mathrm{z}$. The carrier gas was helium $(1.2 \mathrm{~mL} / \mathrm{min})$.

Identification was performed by comparing retention times and mass spectra with those of pure standards, and with mass spectra from NIST05 library. Identification by comparison with mass spectra was considered satisfactory if spectra reverse match numbers (RM) higher than 800 were obtained. If in a particular sample the mass spectra were not clear $(\mathrm{RM}<800)$, identification was considered satisfactory if the ratios of a quantifier and three most abundant characteristic ions reasonably matched those in the reference spectra of a given compound. Linear retention indices (relative to C7-C24 n-alkanes) were calculated and compared to those from literature. When standards were available, standard calibration curves based on quantifier ions were used for quantification. Linearity was satisfactory with coefficient of determination higher than 0.99 for all the standards. 
For other compounds semi-quantitative analysis was carried out, and their concentrations ( $\mu \mathrm{g}$ or $\mathrm{mg} / \mathrm{kg}$ ) were expressed as equivalents of the compounds with similar chemical structure for which standards were available, assuming a response factor equal to one.

\subsection{Analysis of Phenols by UPLC-DAD}

Extraction of phenols from EVOO was performed according to the modified method proposed by Jerman Klen, Golc Wondra, Vrhovšek, and Mozetič Vodopivec [42]. Ten grams of EVOO were dissolved in $10 \mathrm{~mL}$ of $\mathrm{n}$-hexane, and $5 \mathrm{~mL}$ of methanol was added. The mixture was vortexed for $2 \mathrm{~min}$, sonicated for $10 \mathrm{~min}$, and then centrifuged at $5000 \mathrm{rpm}$ for $5 \mathrm{~min}$. The extraction was repeated 2 more times, and unified methanol extracts were defatted by 3 portions of $10 \mathrm{~mL} n$-hexane. The methanol extracts (or standard solutions) were evaporated to dryness, the residue was re-dissolved in a $2 \mathrm{~mL}$ of a mixture of HPLC eluents (A $(95: 5$ water-acetic acid $(v / v)): B($ methanol $)=90: 10(v / v))$, and filtered through $0.45 \mu \mathrm{m}$ PTFE filters.

Analysis of phenols was performed by ultra-performance liquid chromatography with diode array detection (UPLC-DAD) using an Agilent Infinity 1260 system (Agilent Technologies, Palo Alto, CA, USA) equipped with a G1311B quaternary pump, a G1329B autosampler, a G1316A column oven, and a G4212B DAD detector. A Kinetex PFP column $(2.6 \mu \mathrm{m}, 100 \mathrm{~mm} \times 4.6 \mathrm{~mm})$ with a guard was used (Phenomenex, Sydney, Australia) at $27^{\circ} \mathrm{C}$. Solvents were water with glacial acetic acid (95:5, $v / v)(\mathrm{A})$ and methanol (B), with a flow rate of $1 \mathrm{~mL} / \mathrm{min}$. Ten microliters of the extract were injected. A 20-step gradient run used was reported previously [42]. Identification was performed by comparing retention times and UV/Vis spectra with those of pure standards when available, and with UV/Vis spectra from the literature [42]. Detection wavelengths were $280 \mathrm{~nm}$ (for simple phenols, vanillic acid, lignans, and secoiridoids), $320 \mathrm{~nm}$ (vanillin and p-coumaric acid), and $365 \mathrm{~nm}$ (flavonoids), while spectra were registered from 200 to $600 \mathrm{~nm}$. Standard calibration curves were constructed for tyrosol, hydroxytyrosol, vanillic acid, vanillin, $p$-coumaric acid, luteolin, apigenin, pinoresinol, and oleuropein. For other compounds semi-quantitative analysis was carried out: secoiridoids were expressed in $\mathrm{mg} / \mathrm{kg}$ as oleuropein, and acetoxypinoresinol as pinoresinol equivalents, respectively.

\subsection{Statistical Data Elaboration}

Data from GC-IT-MS, UPLC-DAD, and sensory analysis were subjected to one-way analysis of variance (ANOVA), and average values were compared by Least Significant Difference (LSD) test at the level of $p<0.05$. Data were further processed by multivariate techniques, such as forward stepwise linear discriminant analysis (SLDA) and partial least squares discriminant analysis (PLSDA). The main goal of SLDA was to find the most useful variables (volatile compounds) for the mutual differentiation of all the six monovarietal EVOO. SLDA was applied on mean-centered data of a reduced dataset including six groups (varieties) and 50 variables with the highest $F$-ratios obtained in one-way ANOVA. Wilk's lambda was used as a selection criterion with an $F$ statistic factor to establish the significance of the changes in Lambda when a new variable is tested $(F$-value to enter $=1)$. The main goal of PLSDA was to find the most useful variables (volatile compounds) for the differentiation of each of the six investigated monovarietal EVOO from all the other (five) monovarietal EVOOs. For this reason, PLSDA was applied on mean-centered data of six separate datasets each including two groups (a single vs. other five monovarietal EVOOs) and all the 197 variables. Variable Importance in Projection (VIP) scores were determined as the weighted sums of the squares of the weight in the PLSDA. ANOVA and SLDA data elaboration were performed by Statistica v. 13.2 software (StatSoft Inc., Tulsa, OK, USA), while PLSDA analysis was conducted using MetaboAnalyst v. 4.0 (http://www.metaboanalyst.ca) created at the University of Alberta, Canada [43]. 


\section{Results}

\subsection{Volatile Aroma Compound Profiles}

A total of 197 volatile compounds were reported, including 29 hydrocarbons, 29 terpenes, 24 aldehydes, 11 ketones, 23 alcohols, 10 acids, 17 esters, 37 benzenoids, 8 furanoids, and 9 other compounds (Table 1). For many volatiles significant differences between average concentrations in the investigated EVOOs were found. Several volatile compounds emerged as exclusive markers of particular monovarietal EVOOs.

\subsubsection{Hydrocarbons}

Istrian Buža and Rosinjola EVOOs stood out with the highest concentration of particular unsaturated hydrocarbons, such as several non-identified branched-chain alkenes, 3-ethyl-1,5-octadiene and 3,7-decadiene isomers, as well as that of saturated ones, such as decane, undecane, and dodecane (Table 1). On the other hand, lower amounts of the same groups of volatiles were found characteristic for Dalmatian Oblica and Lastovka, while I. bjelica EVOO contained intermediate concentrations. Similar relations were observed when comparing total hydrocarbons. Among hydrocarbons, 2,6-dimethyl-3-heptene turned out to be an exclusive marker of Rosinjola, and dodecene of Lastovka EVOO, respectively.

\subsubsection{Monoterpenes and Sesquiterpenes}

Lastovka EVOO was distinguished by the highest concentrations of several monoterpenes, such as $\alpha$-pinene, camphene, myrcene, $\beta$-phellandrene, and $\gamma$-terpinene, as well as total monoterpenes (Table 1). The same EVOO contained the highest levels of $\gamma$-elemene and particular non-identified sesquiterpenes. Several sesquiterpenes were characteristic for Oblica EVOO, with $\alpha$-muurolene as the most prominent marker. The lowest concentrations of $(+)$-cycloisosativene, $\alpha$-muurolene, $\delta$-cadinene, and two unidentified sesquiterpenes, as well as total sesquiterpenes, were found in Lastovka and Leccino EVOOs.

\subsubsection{Aldehydes}

Among unsaturated aldehydes formed in the so-called LOX pathway, Buža, followed by Rosinjola and Oblica EVOOs, contained the highest concentrations of (E)- and (Z)-3-hexenal, respectively (Table 1). The same monovarietal EVOOs, with sporadic exceptions, were also distinguished by high levels of pentenals, hexadienals, and (E)-2-octenal. Oblica EVOO had high concentration of decadienals. Leccino was clearly distinguished from the other monovarietal EVOOs by the highest level of the major EVOO volatile, $(E)$-2-hexenal, as well as that of $(E, E)-2,4$-heptadienal. Leccino EVOO had the lowest concentration of (Z)-3-hexenal, although not statistically different from that found in I. bjelica and Lastovka EVOOs. Lastovka EVOO was characterized by rather low levels of particular LOX-derived hexenals, as well as pentenals, octanal, and hexadienals. Istarska bjelica contained low concentrations of (Z)-3-hexenal and decadienals. Among saturated aldehydes originating from the processes other than LOX, 3-methylbutanal turned out to be an exclusive marker of I. bjelica, while abundance in 2-methyl-2-pentenal was observed in Rosinjola EVOO (Table 1). Higher levels of hexanal clearly discriminated Dalmatian (Oblica and Lastovka) from Istrian monovarietal EVOOs. 
Table 1. Concentrations ( $\mu \mathrm{g} / \mathrm{kg}$ unless otherwise stated) of volatile aroma compounds determined by gas chromatography-ion trap mass spectrometry (GC-IT-MS) after headspace solid-phase microextraction (HS-SPME) from monovarietal extra virgin olive oils produced from Buža, Istarska bjelica, Rosinjola, Oblica, Lastovka, and Leccino varieties in Croatia.

\begin{tabular}{|c|c|c|c|c|c|c|c|c|}
\hline \multirow{2}{*}{ Compound } & \multirow{2}{*}{ ID } & \multirow{2}{*}{ LRI } & \multicolumn{6}{|c|}{ Variety } \\
\hline & & & Buža & I. bjelica & Rosinjola & Oblica & Lastovka & Leccino \\
\hline hydrocarbons & & & & & & & & \\
\hline propane & MS & $<700$ & $6.64^{b c}$ & $14.91^{\mathrm{a}}$ & $1.12^{\mathrm{c}}$ & $11.49^{a b}$ & $12.51^{\mathrm{a}}$ & $5.43^{c}$ \\
\hline (Z)-2-pentene & MS & $<700$ & $61.19^{b}$ & $80.27^{a}$ & $60.31^{b}$ & $33.98^{c}$ & $43.63^{c}$ & $83.09^{\mathrm{a}}$ \\
\hline 1,4 -pentadiene $(\mathrm{mg} / \mathrm{kg})$ & LRI,MS & $<700$ & $0.17^{\mathrm{a}}$ & $0.16^{\mathrm{a}}$ & $0.15^{\mathrm{ab}}$ & $0.10^{c}$ & $0.09^{c}$ & $0.12^{b}$ \\
\hline 1,3-pentadiene & LRI,MS & $<700$ & $81.73^{a}$ & $75.36^{\mathrm{ab}}$ & $71.98^{a b}$ & $53.80^{c}$ & $48.98^{c}$ & $62.92 \mathrm{bc}$ \\
\hline 1-heptene & LRI,MS & 731 & 5.42 & 16.64 & 3.25 & 9.76 & 5.72 & 6.13 \\
\hline 3,5-dimethylheptane & MS & 753 & 1.61 & 3.59 & 3.63 & 2.38 & 0.33 & 2.86 \\
\hline propanal & LRI,MS & 778 & 7.00 & 5.23 & 7.36 & 5.10 & 5.68 & 7.04 \\
\hline octane & S,LRI,MS & 800 & 31.03 & 35.33 & 27.29 & 30.78 & 40.92 & 27.11 \\
\hline 2,6-dimethyl-3-heptene & MS & 821 & $0.20^{\mathrm{b}}$ & $0.58^{\mathrm{b}}$ & $6.17^{\mathrm{a}}$ & $0.00^{\mathrm{b}}$ & $0.00^{\mathrm{b}}$ & $0.19^{\mathrm{b}}$ \\
\hline$(E)$-2-octene & LRI,MS & 832 & 3.50 & 4.60 & 3.81 & 3.36 & 5.62 & 3.60 \\
\hline branched-chain alkene I (n.i.) & MS & 953 & $94.32^{\mathrm{a}}$ & $63.73^{c}$ & $85.98^{a b}$ & $47.29^{d}$ & $38.96^{d}$ & $74.97^{\mathrm{bc}}$ \\
\hline branched-chain alkene II (n.i.) & MS & 960 & $84.13^{\mathrm{a}}$ & $56.70^{c}$ & $71.63^{\mathrm{b}}$ & $41.67^{\mathrm{d}}$ & $35.22^{d}$ & $65.24^{b c}$ \\
\hline decane & S,LRI,MS & 997 & $31.53^{a b}$ & $19.98 \mathrm{bc}$ & $39.92^{\mathrm{a}}$ & $9.49^{c}$ & $7.39^{\mathrm{c}}$ & $14.67^{c}$ \\
\hline 3-ethyl-1,5-octadiene I (mg/kg) & LRI,MS & 1006 & $0.36^{\mathrm{a}}$ & $0.26^{\mathrm{c}}$ & $0.34 \mathrm{ab}$ & $0.18^{\mathrm{d}}$ & $0.15^{\mathrm{d}}$ & $0.31^{\mathrm{b}}$ \\
\hline 3-ethyl-1,5-octadiene II (mg/kg) & LRI,MS & 1020 & $0.40^{\mathrm{a}}$ & $0.25^{c}$ & $0.35^{\mathrm{ab}}$ & $0.20^{\mathrm{d}}$ & $0.16^{\mathrm{d}}$ & $0.31^{\mathrm{b}}$ \\
\hline 3-ethyl-1,5-octadiene III & LRI,MS & 1033 & 2.99 & 12.91 & 3.19 & 1.24 & 0.79 & 2.60 \\
\hline branched-chain alkene III (n.i.) & MS & 1067 & 0.37 & 10.08 & 0.45 & 0.06 & 0.01 & 0.48 \\
\hline 3,7-decadiene I & LRI,MS & 1074 & $76.81^{\mathrm{a}}$ & $44.98^{c}$ & $71.69^{a b}$ & $33.06^{\mathrm{d}}$ & $29.07^{d}$ & $65.14^{\mathrm{b}}$ \\
\hline branched-chain alkene IV (n.i.) & MS & 1077 & 3.59 & 6.51 & 1.87 & 1.62 & 0.72 & 2.92 \\
\hline 3,7-decadiene II (mg/kg) & LRI,MS & 1080 & $0.29^{a}$ & $0.17^{\mathrm{b}}$ & $0.28^{a}$ & $0.15^{b c}$ & $0.13^{c}$ & $0.26^{\mathrm{a}}$ \\
\hline 3,7-decadiene III (mg/kg) & LRI,MS & 1084 & $0.29^{a}$ & $0.15^{\mathrm{c}}$ & $0.25^{\mathrm{ab}}$ & $0.15^{c}$ & $0.11^{\mathrm{c}}$ & $0.21^{b}$ \\
\hline branched-chain alkene $V$ (n.i.) & MS & 1087 & $7.98^{a b}$ & $11.87^{\mathrm{a}}$ & $6.30^{\mathrm{ab}}$ & $4.05^{\mathrm{b}}$ & $3.00^{b}$ & $5.80^{\mathrm{b}}$ \\
\hline undecane & S,LRI,MS & 1095 & $18.56^{\mathrm{ab}}$ & $9.75^{b}$ & $39.12^{\mathrm{a}}$ & $3.29^{b}$ & $2.09^{b}$ & $9.83^{b}$ \\
\hline dodecane & S,LRI,MS & 1203 & $23.4^{\mathrm{ab}}$ & $14.16^{\mathrm{bc}}$ & $35.51^{\mathrm{a}}$ & $4.64^{\mathrm{c}}$ & $2.64^{\mathrm{c}}$ & $13.66^{\mathrm{bc}}$ \\
\hline dodecene & LRI,MS & 1241 & $27.26^{b c}$ & $33.95^{b}$ & $17.25^{\mathrm{cd}}$ & $14.74^{\mathrm{cd}}$ & $58.52^{\mathrm{a}}$ & $8.43^{\mathrm{d}}$ \\
\hline 3-propylcyclohexene & MS & 1247 & $10.97 \mathrm{bc}$ & $7.93^{c}$ & $14.15^{\mathrm{ab}}$ & $13.41^{\mathrm{ab}}$ & $9.90^{b c}$ & $14.85^{\mathrm{a}}$ \\
\hline 1,5,5,6-tetramethyl-1,3-cyclohexadiene & LRI,MS & 1365 & $2.46^{\mathrm{bc}}$ & $1.55^{\mathrm{c}}$ & $4.38^{\mathrm{abc}}$ & $5.73^{\mathrm{a}}$ & $5.52^{\mathrm{ab}}$ & $1.82^{\mathrm{c}}$ \\
\hline 2,2-dimethyl-(Z)-3-hexene I & MS & 1499 & $28.41^{\mathrm{a}}$ & $16.09^{b}$ & $33.60^{\mathrm{a}}$ & $26.67^{\mathrm{a}}$ & $14.60^{\mathrm{b}}$ & $10.76^{\mathrm{b}}$ \\
\hline 2,2-dimethyl-(Z)-3-hexene II & MS & 1560 & 4.79 & 2.12 & 6.05 & 4.78 & 2.39 & 2.88 \\
\hline
\end{tabular}


Table 1. Cont

\begin{tabular}{|c|c|c|c|c|c|c|c|c|}
\hline \multirow{2}{*}{ Compound } & \multirow{2}{*}{ ID } & \multirow{2}{*}{ LRI } & \multicolumn{6}{|c|}{ Variety } \\
\hline & & & Buža & I. bjelica & Rosinjola & Oblica & Lastovka & Leccino \\
\hline \multicolumn{9}{|l|}{ monoterpenes } \\
\hline$\alpha$-pinene & S,LRI,MS & 1015 & $6.28^{b}$ & $4.91^{b}$ & $12.25^{b}$ & $8.90^{b}$ & $41.71^{\mathrm{a}}$ & $2.79 \mathrm{~b}$ \\
\hline camphene & S,LRI,MS & 1053 & $0.23^{b}$ & $0.10^{b}$ & $0.25^{b}$ & $0.31^{b}$ & $1.35^{\mathrm{a}}$ & $0.04^{b}$ \\
\hline$\beta$-pinene & S,LRI,MS & 1099 & 0.41 & 0.77 & 1.98 & 1.10 & 83.74 & 0.46 \\
\hline sabinene & S,LRI,MS & 1112 & 2.07 & 0.79 & 2.09 & 2.94 & 49.44 & 17.20 \\
\hline 3-carene & S,LRI,MS & 1139 & $4.72^{\mathrm{a}}$ & $3.06^{\mathrm{b}}$ & $4.20^{a b}$ & $0.97^{\mathrm{c}}$ & $2.29 \mathrm{bc}$ & $0.83^{c}$ \\
\hline monoterpene I (n.i.) & MS & 1140 & $2.77^{\mathrm{a}}$ & $1.84^{\mathrm{b}}$ & $2.54^{\mathrm{ab}}$ & $0.40^{c}$ & $1.35^{b c}$ & $0.27^{c}$ \\
\hline myrcene & S,LRI,MS & 1157 & $8.60^{b}$ & $4.36^{\mathrm{b}}$ & $19.76^{a b}$ & $11.48^{\mathrm{b}}$ & $41.67^{\mathrm{a}}$ & $11.85^{\mathrm{b}}$ \\
\hline$\alpha$-terpinene & S,LRI,MS & 1171 & 0.37 & 0.23 & 1.12 & 0.72 & 10.84 & 9.91 \\
\hline limonene $(\mathrm{mg} / \mathrm{kg})$ & S,LRI,MS & 1191 & 0.02 & 0.08 & 0.02 & 0.02 & 0.64 & 0.01 \\
\hline$\beta$-phellandrene & LRI,MS & 1201 & $0.00^{\mathrm{b}}$ & $0.00^{b}$ & $0.00^{b}$ & $0.03^{\mathrm{b}}$ & $4.25^{\mathrm{a}}$ & $0.02^{b}$ \\
\hline$(Z)$-ocimene & S,LRI,MS & 1230 & $15.14^{\mathrm{b}}$ & $8.00^{\mathrm{b}}$ & $25.27^{a b}$ & $34.84^{\mathrm{a}}$ & $37.26^{\mathrm{a}}$ & $10.72^{b}$ \\
\hline$\gamma$-terpinene & S,LRI,MS & 1238 & $30.50^{b}$ & $4.96^{\mathrm{b}}$ & $4.17^{\mathrm{b}}$ & $3.74^{\mathrm{b}}$ & $436.30^{\mathrm{a}}$ & $40.80^{\mathrm{b}}$ \\
\hline (E)-ocimene (mg/kg) & S,LRI,MS & 1245 & $0.21^{b c}$ & $0.09^{c}$ & $0.27 \mathrm{abc}$ & $0.52^{\mathrm{a}}$ & $0.43^{\mathrm{ab}}$ & $0.12^{c}$ \\
\hline monoterpene II (n.i.) & MS & 1260 & $0.69 \mathrm{bc}$ & $0.40^{\mathrm{c}}$ & $1.28^{a b c}$ & $1.82^{\mathrm{a}}$ & $1.49 \mathrm{ab}$ & $0.53 \mathrm{bc}$ \\
\hline terpinolene & S,LRI,MS & 1275 & 0.44 & 0.28 & 0.70 & 0.38 & 3.83 & 0.84 \\
\hline (Z)-alloocimene & LRI,MS & 1349 & 11.38 & 9.68 & 10.20 & 23.02 & 8.22 & 7.20 \\
\hline (E)-alloocimene & S,LRI,MS & 1357 & 16.79 & 12.63 & 13.25 & 37.22 & 10.66 & 9.26 \\
\hline \multicolumn{8}{|l|}{ sesquiterpenes } & $22.04^{a b c}$ \\
\hline$(+)$-cycloisosativene $(\mathrm{mg} / \mathrm{kg})$ & LRI,MS & 1477 & $1.15^{\mathrm{a}}$ & $0.87^{b}$ & $0.80^{\mathrm{b}}$ & $0.89^{a b}$ & $0.24^{c}$ & $0.26^{c}$ \\
\hline$\alpha$-copaene $(\mathrm{mg} / \mathrm{kg})$ & LRI,MS & 1487 & $8.44^{\mathrm{a}}$ & $7.02^{a b}$ & $5.81^{b}$ & $8.77^{\mathrm{a}}$ & $2.14^{\mathrm{c}}$ & $1.64^{\mathrm{c}}$ \\
\hline sesquiterpene I (n.i.) & MS & 1536 & $0.00^{\mathrm{b}}$ & $0.00^{b}$ & $0.00^{\mathrm{b}}$ & $0.00^{\mathrm{b}}$ & $3.44^{\mathrm{a}}$ & $0.00^{b}$ \\
\hline sesquiterpene II (n.i.) (mg/kg) & MS & 1583 & $0.29^{\mathrm{a}}$ & $0.27^{\mathrm{a}}$ & $0.23^{a}$ & $0.28^{\mathrm{a}}$ & $0.08^{b}$ & $0.05^{b}$ \\
\hline sesquiterpene III (n.i.) (mg/kg) & MS & 1683 & $0.11^{\mathrm{ab}}$ & $0.09^{b}$ & $0.09^{b}$ & $0.13^{a}$ & $0.03^{c}$ & $0.02^{c}$ \\
\hline$\delta$-selinene & LRI,MS & 1698 & $24.52^{b}$ & $25.84^{\mathrm{ab}}$ & $10.32^{\mathrm{c}}$ & $32.29^{a}$ & $6.82^{c}$ & $2.07^{\mathrm{c}}$ \\
\hline$\gamma$-elemene $(\mathrm{mg} / \mathrm{kg})$ & MS & 1704 & $0.04^{\mathrm{b}}$ & $0.05^{\mathrm{b}}$ & $0.05^{\mathrm{b}}$ & $0.06^{\mathrm{b}}$ & $0.20^{\mathrm{a}}$ & $0.05^{b}$ \\
\hline$\alpha$-muurolene $(\mathrm{mg} / \mathrm{kg})$ & LRI,MS & 1719 & $1.65^{b}$ & $1.42^{b}$ & $1.26^{\mathrm{b}}$ & $2.10^{\mathrm{a}}$ & $0.49^{c}$ & $0.34^{\mathrm{c}}$ \\
\hline sesquiterpene IV (n.i.) & MS & 1736 & $0.63^{c}$ & $3.02^{c}$ & $26.21^{b}$ & $2.33^{c}$ & $51.54^{\mathrm{a}}$ & $29.75^{b}$ \\
\hline$\alpha$-farnesene $(\mathrm{mg} / \mathrm{kg})$ & LRI,MS & 1745 & $0.53^{a}$ & $0.29^{b}$ & $0.84^{\mathrm{a}}$ & $0.12^{b}$ & $0.25^{b}$ & $0.17^{\mathrm{b}}$ \\
\hline $\begin{array}{c}\delta \text {-cadinene }(\mathrm{mg} / \mathrm{kg}) \\
\text { aldehydes }\end{array}$ & LRI,MS & 1750 & $0.14^{\mathrm{ab}}$ & $0.11^{b}$ & $0.17^{\mathrm{a}}$ & $0.17^{\mathrm{a}}$ & $0.06^{\mathrm{c}}$ & $0.07^{c}$ \\
\hline acrolein & LRI,MS & 829 & $6.07^{\mathrm{a}}$ & $2.34^{\mathrm{d}}$ & $4.66^{\mathrm{ab}}$ & $4.23 \mathrm{bc}$ & $2.60^{\mathrm{cd}}$ & $3.75^{b c}$ \\
\hline
\end{tabular}


Table 1. Cont

\begin{tabular}{|c|c|c|c|c|c|c|c|c|}
\hline \multirow{2}{*}{ Compound } & \multirow{2}{*}{ ID } & \multirow{2}{*}{ LRI } & \multicolumn{6}{|c|}{ Variety } \\
\hline & & & Buža & I. bjelica & Rosinjola & Oblica & Lastovka & Leccino \\
\hline 2-methylbutanal & LRI,MS & 903 & 10.74 & 13.39 & 11.93 & 7.86 & 18.70 & 13.76 \\
\hline 3-methylbutanal & S,LRI,MS & 906 & $10.51^{b}$ & $22.29^{a}$ & $10.65^{b}$ & $8.49^{\mathrm{b}}$ & $12.31^{b}$ & $12.26^{\mathrm{b}}$ \\
\hline 2-methyl-2-pentenal & MS & 930 & $1.08^{c}$ & $0.26^{\mathrm{cd}}$ & $3.34^{\mathrm{a}}$ & $2.15^{b}$ & $0.85^{\mathrm{cd}}$ & $0.00^{\mathrm{d}}$ \\
\hline (E)-2-butenal & S,LRI,MS & 1024 & 11.44 & 9.98 & 12.87 & 9.10 & 18.39 & 25.45 \\
\hline unsaturated aliphatic aldehyde I (n.i.) & MS & 1036 & $3.27^{\mathrm{a}}$ & $2.25^{\mathrm{b}}$ & $2.63^{\mathrm{ab}}$ & $2.48^{\mathrm{ab}}$ & $1.56^{\mathrm{b}}$ & $1.68^{\mathrm{b}}$ \\
\hline unsaturated aliphatic aldehyde II (n.i.) & MS & 1051 & 0.25 & 5.31 & 0.55 & 0.03 & 0.04 & 0.25 \\
\hline hexanal $(\mathrm{mg} / \mathrm{kg})$ & S,LRI,MS & 1070 & $0.24^{b}$ & $0.29^{b}$ & $0.27^{\mathrm{b}}$ & $0.44^{\mathrm{a}}$ & $0.53^{\mathrm{a}}$ & $0.20^{\mathrm{b}}$ \\
\hline$(Z)$-2-pentenal & LRI,MS & 1093 & $11.73^{a b}$ & $7.43^{c}$ & $16.92^{\text {a }}$ & $8.09 \mathrm{bc}$ & $5.29^{c}$ & $5.70^{c}$ \\
\hline (E)-2-pentenal & S,LRI,MS & 1115 & $52.30^{\mathrm{a}}$ & $48.87^{\mathrm{a}}$ & $43.67^{\mathrm{ab}}$ & $39.40^{\mathrm{ab}}$ & $24.74^{b}$ & $42.38^{\mathrm{ab}}$ \\
\hline (E)-3-hexenal & LRI,MS & 1125 & $57.10^{\mathrm{a}}$ & $42.81^{b}$ & $67.22^{\mathrm{a}}$ & $43.82^{a b}$ & $23.48^{c}$ & $35.30^{\mathrm{bc}}$ \\
\hline (Z)-3-hexenal (mg/kg) & LRI,MS & 1130 & $1.36^{\mathrm{a}}$ & $0.40^{\mathrm{cd}}$ & $0.96^{\mathrm{abc}}$ & $1.09 \mathrm{ab}$ & $0.47^{b c d}$ & $0.14^{\mathrm{d}}$ \\
\hline heptanal & LRI,MS & 1175 & 4.37 & 3.60 & 4.33 & 3.19 & 3.03 & 3.90 \\
\hline (Z)-2-hexenal & LRI,MS & 1189 & 44.49 & 31.63 & 48.50 & 33.38 & 39.16 & 32.68 \\
\hline (E)-2-hexenal (mg/kg) & S,LRI,MS & 1205 & $19.38^{b c}$ & $22.54^{\mathrm{b}}$ & $21.96^{b}$ & $11.90 \mathrm{~cd}$ & $5.92^{\mathrm{d}}$ & $34.95^{\mathrm{a}}$ \\
\hline octanal $(\mathrm{mg} / \mathrm{kg})$ & S,LRI,MS & 1280 & $0.10^{\mathrm{a}}$ & $0.11^{\mathrm{a}}$ & $0.09^{a}$ & $0.07^{b}$ & $0.07^{b}$ & $0.09^{a}$ \\
\hline (Z)-2-heptenal & LRI,MS & 1312 & 10.60 & 7.65 & 4.80 & 11.30 & 7.66 & 7.12 \\
\hline$(E, E)-2,4$-hexadienal $(\mathrm{mg} / \mathrm{kg})$ & LRI,MS & 1381 & $0.27 \mathrm{ab}$ & $0.16^{\mathrm{cd}}$ & $0.35^{\mathrm{a}}$ & $0.20 \mathrm{bc}$ & $0.07^{\mathrm{d}}$ & $0.13^{\mathrm{cd}}$ \\
\hline$(E, Z)-2,4$-hexadienal $(\mathrm{mg} / \mathrm{kg})$ & LRI,MS & 1385 & $1.56^{\mathrm{ab}}$ & $1.06^{\mathrm{bc}}$ & $2.19^{a}$ & $0.87^{\mathrm{cd}}$ & $0.38^{\mathrm{d}}$ & $0.62^{\mathrm{cd}}$ \\
\hline$(E)$-2-octenal & S,LRI,MS & 1417 & $11.75^{\mathrm{a}}$ & $5.53^{c}$ & $8.12^{b c}$ & $10.47^{\mathrm{ab}}$ & $7.17^{b c}$ & $9.15^{b}$ \\
\hline$(E, E)-2,4$-heptadienal & LRI,MS & 1448 & $33.23^{\mathrm{b}}$ & $19.61^{\mathrm{d}}$ & 30.68 bcd & $32.10^{b c}$ & $21.83^{c}$ & $44.72^{\mathrm{a}}$ \\
\hline 2-isopropylidene-3-methylhexa-3,5-dienal & MS & 1460 & $0.36^{\mathrm{bc}}$ & $0.33^{c}$ & $0.65^{\mathrm{ab}}$ & $0.11^{\mathrm{c}}$ & $0.09^{c}$ & $0.70^{\mathrm{a}}$ \\
\hline$(E, E)$-2,4-decadienal & LRI,MS & 1750 & $0.65^{b c}$ & $0.49^{\mathrm{c}}$ & $1.06^{\mathrm{ab}}$ & $1.52^{\mathrm{a}}$ & $0.85^{b}$ & $0.85^{\mathrm{b}}$ \\
\hline$(E, Z)$-2,4-decadienal & LRI,MS & 1794 & $0.67^{\mathrm{b}}$ & $0.26^{c}$ & $0.47^{b c}$ & $1.13^{\mathrm{a}}$ & $0.93^{a b}$ & $0.61^{b c}$ \\
\hline $\begin{array}{l}\text { tetradecanal } \\
\text { ketones }\end{array}$ & LRI,MS & 1909 & 1.05 & 0.97 & 1.04 & 1.41 & 0.99 & 0.62 \\
\hline 3-pentanone $(\mathrm{mg} / \mathrm{kg})$ & LRI,MS & 962 & $0.09^{c}$ & $0.23^{\mathrm{a}}$ & $0.09^{c}$ & $0.13^{b c}$ & $0.19^{a b}$ & $0.08^{c}$ \\
\hline 1-penten-3-one (mg/kg) & S,LRI,MS & 1008 & $0.29^{a}$ & $0.29^{a}$ & $0.26^{\mathrm{ab}}$ & $0.18^{\mathrm{bc}}$ & $0.12^{\mathrm{c}}$ & $0.22^{\mathrm{ab}}$ \\
\hline 3-hexen-2-one & MS & 1121 & $0.57^{\mathrm{b}}$ & $0.67^{\mathrm{a}}$ & $0.58^{\mathrm{b}}$ & $0.44^{c}$ & $0.55^{b}$ & $0.45^{c}$ \\
\hline 2-octanone & LRI,MS & 1275 & 6.12 & 0.62 & 0.54 & 1.55 & 1.51 & 0.89 \\
\hline 2-methyl-6-methylene-1,7-octadien-3-one & LRI,MS & 1303 & $44.85^{\mathrm{a}}$ & $21.50^{\mathrm{b}}$ & $51.45^{\mathrm{a}}$ & $24.25^{b}$ & $16.32^{b}$ & $62.59^{\mathrm{a}}$ \\
\hline 6-methyl-5-hepten-2-one & LRI,MS & 1326 & $2.15^{b c}$ & $1.94^{\mathrm{bc}}$ & $2.63^{a b c}$ & $3.02^{\mathrm{ab}}$ & $4.40^{\mathrm{a}}$ & $1.40^{\mathrm{c}}$ \\
\hline 2-cyclohexene-1,4-dione I & MS & 1712 & $3.62^{\mathrm{ab}}$ & $2.27^{\mathrm{cd}}$ & $5.11^{\mathrm{a}}$ & $3.20 \mathrm{bc}$ & $2.05^{\mathrm{bcd}}$ & $1.04^{\mathrm{d}}$ \\
\hline 2-cyclohexene-1,4-dione II & MS & 1803 & $1.15^{\mathrm{ab}}$ & $0.68^{\mathrm{cd}}$ & $1.57^{\mathrm{a}}$ & $1.06^{\mathrm{abc}}$ & $0.64^{\mathrm{cd}}$ & $0.29 \mathrm{~d}$ \\
\hline
\end{tabular}


Table 1. Cont

\begin{tabular}{|c|c|c|c|c|c|c|c|c|}
\hline \multirow{2}{*}{ Compound } & \multirow{2}{*}{ ID } & \multirow{2}{*}{ LRI } & \multicolumn{6}{|c|}{ Variety } \\
\hline & & & Buža & I. bjelica & Rosinjola & Oblica & Lastovka & Leccino \\
\hline (Z)-cinerolone & MS & 2002 & $0.20^{\mathrm{b}}$ & $0.13^{c}$ & $0.19^{b}$ & $0.04^{\mathrm{d}}$ & $0.03^{\mathrm{d}}$ & $0.28^{\mathrm{a}}$ \\
\hline 1-(2,6,6-trimethyl-1-cyclohexen-1-yl)-1-penten-3-one & MS & 2056 & $0.18^{c}$ & $0.25 \mathrm{bc}$ & $0.14^{\mathrm{c}}$ & $0.51^{\mathrm{a}}$ & $0.47^{a b}$ & $0.12^{\mathrm{c}}$ \\
\hline $\begin{array}{l}4^{\prime} \text {-ethoxy-2'-hydroxyoctadecanophenone } \\
\text { alcohols }\end{array}$ & MS & 2081 & $0.26^{\mathrm{bc}}$ & $0.12^{\mathrm{c}}$ & $0.90^{\mathrm{a}}$ & $0.24^{\mathrm{bc}}$ & $0.26^{\mathrm{bc}}$ & $0.41^{\mathrm{b}}$ \\
\hline 2-methyl-2-propanol & LRI,MS & 886 & 4.98 & 4.93 & 8.45 & 0.03 & 0.00 & 4.33 \\
\hline 1-methoxy-2-propanol (mg/kg) & MS & 917 & $0.15^{\mathrm{b}}$ & $0.12^{b}$ & $0.18^{\mathrm{ab}}$ & $0.15^{b}$ & $0.23^{a}$ & $0.14^{\mathrm{b}}$ \\
\hline 3-pentanol & LRI,MS & 1097 & 11.84 & 16.51 & 6.53 & 6.20 & 15.09 & 5.92 \\
\hline 2-pentanol & LRI,MS & 1109 & 1.04 & 2.75 & 0.67 & 2.76 & 1.78 & 1.59 \\
\hline 1-penten-3-ol $(\mathrm{mg} / \mathrm{kg})$ & LRI,MS & 1148 & $0.18^{\mathrm{b}}$ & $0.28^{\mathrm{a}}$ & $0.19^{b}$ & $0.14^{\mathrm{b}}$ & $0.19^{\mathrm{b}}$ & $0.18^{\mathrm{b}}$ \\
\hline 3-methyl-1-butanol & S,LRI,MS & 1197 & $19.96^{\mathrm{b}}$ & $26.70^{b}$ & $22.74^{b}$ & $27.21^{b}$ & $57.71^{\text {a }}$ & $23.80^{\mathrm{b}}$ \\
\hline 1-pentanol & S,LRI,MS & 1239 & $10.37^{b c}$ & $16.24^{\mathrm{ab}}$ & $7.86^{\mathrm{bc}}$ & $20.10^{\mathrm{a}}$ & $22.07^{\mathrm{a}}$ & $6.96^{\mathrm{c}}$ \\
\hline (E)-2-penten-1-ol & S,LRI,MS & 1300 & 62.07 & 65.28 & 64.27 & 43.81 & 67.92 & 61.24 \\
\hline (Z)-2-penten-1-ol (mg/kg) & S,LRI,MS & 1308 & $0.26^{\mathrm{a}}$ & $0.31^{\mathrm{a}}$ & $0.27^{\mathrm{a}}$ & $0.21^{b}$ & $0.28^{\mathrm{a}}$ & $0.25^{\mathrm{ab}}$ \\
\hline 1-hexanol $(\mathrm{mg} / \mathrm{kg})$ & S,LRI,MS & 1342 & 0.70 & 1.21 & 1.20 & 1.17 & 1.58 & 0.76 \\
\hline (E)-3-hexen-1-ol & LRI,MS & 1352 & $27.42^{\mathrm{ab}}$ & $27.45^{\mathrm{ab}}$ & $27.49 \mathrm{ab}$ & $37.90^{\mathrm{a}}$ & $46.03^{a}$ & $14.76^{\mathrm{b}}$ \\
\hline (Z)-3-hexen-1-ol (mg/kg) & S,LRI,MS & 1372 & $1.63^{\mathrm{b}}$ & $1.08^{b c}$ & $1.50 \mathrm{bc}$ & $2.77^{a}$ & $1.92 \mathrm{ab}$ & $0.61^{\mathrm{c}}$ \\
\hline (E)-2-hexen-1-ol (mg/kg) & S,LRI,MS & 1394 & $1.00^{b}$ & $1.68^{\mathrm{b}}$ & $1.37^{\mathrm{b}}$ & $1.05^{b}$ & $3.42^{\mathrm{a}}$ & $1.42^{b}$ \\
\hline (Z)-2-hexen-1-ol & S,LRI,MS & 1402 & 7.53 & 122.27 & 11.84 & 11.20 & 15.53 & 10.63 \\
\hline 1-heptanol & S,LRI,MS & 1445 & 4.05 & 2.16 & 2.23 & 3.38 & 3.39 & 2.71 \\
\hline 2-ethyl-1-hexanol & S,LRI,MS & 1480 & $4.58^{\mathrm{d}}$ & $5.90^{c}$ & $10.39^{a}$ & $4.32^{\mathrm{d}}$ & $4.01^{\mathrm{d}}$ & $6.93^{b}$ \\
\hline 1-octanol & S,LRI,MS & 1546 & 7.65 & 5.29 & 5.41 & 5.56 & 6.01 & 5.98 \\
\hline 2,4-hexadien-1-ol & LRI,MS & 1568 & 0.11 & 0.10 & 0.00 & 0.65 & 0.64 & 0.00 \\
\hline 1-nonanol & S,LRI,MS & 1650 & 3.73 & 2.56 & 3.14 & 2.53 & 3.32 & 3.10 \\
\hline 3-methyl-3-cyclohexen-1-ol & MS & 1744 & $4.66^{\mathrm{a}}$ & $2.34^{b}$ & $4.39^{\mathrm{a}}$ & $2.25^{b}$ & $0.65^{b}$ & $0.88^{b}$ \\
\hline 2-(2-butoxyethoxy)-ethanol & LRI,MS & 1779 & $3.83^{b}$ & $2.43^{b}$ & $42.91^{a}$ & $7.86^{b}$ & $7.62^{b}$ & $9.91^{b}$ \\
\hline 2,2'-oxybis-1-propanol & LRI,MS & 1865 & 4.04 & 3.46 & 4.51 & 2.27 & 2.59 & 2.44 \\
\hline $\begin{array}{l}\text { tetradecanol } \\
\text { acids }\end{array}$ & LRI,MS & 2158 & $25.23^{\mathrm{a}}$ & $26.72^{\mathrm{a}}$ & $12.11^{\mathrm{ab}}$ & $31.35^{\mathrm{a}}$ & $13.64^{\mathrm{ab}}$ & $6.70^{\mathrm{b}}$ \\
\hline acetic acid (mg/kg) & S,LRI,MS & 1427 & 1.82 & 2.55 & 2.46 & 2.49 & 4.53 & 1.70 \\
\hline acid (n.i.) (mg/kg) & MS & 1476 & $0.91^{\mathrm{ab}}$ & $0.82^{b}$ & $1.17^{\mathrm{a}}$ & $1.10^{\mathrm{a}}$ & $1.18^{\mathrm{a}}$ & $1.07^{\mathrm{a}}$ \\
\hline butanoic acid & S,LRI,MS & 1602 & $27.65^{c}$ & $41.57^{b c}$ & $40.46^{b c}$ & $52.13^{\mathrm{ab}}$ & $72.47^{a}$ & $30.43^{b c}$ \\
\hline hexanoic acid (mg/kg) & S,LRI,MS & 1823 & $0.76^{c}$ & $1.23 \mathrm{bc}$ & $1.32^{b c}$ & $1.74^{\mathrm{ab}}$ & $2.21^{\mathrm{a}}$ & $1.00^{c}$ \\
\hline 2-ethylhexanoic acid & LRI,MS & 1925 & $3.41^{\mathrm{c}}$ & $8.59^{c}$ & $52.77^{\text {a }}$ & $10.47^{b c}$ & $12.29 \mathrm{bc}$ & $23.46^{\mathrm{b}}$ \\
\hline (E)-3-hexenoic acid & LRI,MS & 1926 & $12.93^{b}$ & $9.33 \mathrm{bc}$ & $16.87^{a b}$ & $24.46^{\mathrm{a}}$ & $5.08^{b c}$ & $1.38^{\mathrm{c}}$ \\
\hline
\end{tabular}


Table 1. Cont

\begin{tabular}{|c|c|c|c|c|c|c|c|c|}
\hline \multirow{2}{*}{ Compound } & \multirow{2}{*}{ ID } & \multirow{2}{*}{ LRI } & \multicolumn{6}{|c|}{ Variety } \\
\hline & & & Buža & I. bjelica & Rosinjola & Oblica & Lastovka & Leccinc \\
\hline octanoic acid $(\mathrm{mg} / \mathrm{kg})$ & S,LRI,MS & 2033 & $0.48^{b}$ & $7.82^{\mathrm{a}}$ & $1.29^{b}$ & $1.65^{\mathrm{b}}$ & $1.47^{b}$ & $0.59^{b}$ \\
\hline sorbic acid & LRI,MS & 2048 & $7.83^{b}$ & $4.94^{b c}$ & $14.78^{\mathrm{a}}$ & $8.44^{\mathrm{b}}$ & $4.58^{b c}$ & $1.04^{\mathrm{c}}$ \\
\hline nonanoic acid $(\mathrm{mg} / \mathrm{kg})$ & S,LRI,MS & 2139 & $41.76^{\mathrm{b}}$ & $434.34^{\mathrm{a}}$ & $54.30^{\mathrm{b}}$ & $41.10^{\mathrm{b}}$ & $55.83^{b}$ & $29.51^{b}$ \\
\hline $\begin{array}{c}\text { decanoic acid }(\mathrm{mg} / \mathrm{kg}) \\
\text { esters }\end{array}$ & S,LRI,MS & 2244 & $0.07^{\mathrm{b}}$ & $0.43^{\mathrm{a}}$ & $0.14^{\mathrm{b}}$ & $0.02^{b}$ & $0.06^{\mathrm{b}}$ & $0.06^{\mathrm{b}}$ \\
\hline allyl acetate & LRI,MS & 805 & 53.61 & 58.55 & 69.96 & 43.21 & 30.69 & 37.81 \\
\hline methyl acetate & LRI,MS & 816 & $91.00^{b}$ & $166.56^{\mathrm{a}}$ & $101.38^{\mathrm{ab}}$ & $59.88^{b}$ & $115.21^{\mathrm{ab}}$ & $81.99^{b}$ \\
\hline 1,1,1-trimethoxyethane & MS & 871 & $61.25^{\mathrm{b}}$ & $328.53^{a}$ & $117.33^{a b}$ & $50.70^{\mathrm{b}}$ & $85.45^{b}$ & $24.70^{b}$ \\
\hline ethyl 2-methylbutanoate & LRI,MS & 1042 & 0.90 & 0.64 & 0.55 & 0.70 & 0.70 & 0.51 \\
\hline isoamyl acetate & S,LRI,MS & 1114 & $4.91^{\mathrm{a}}$ & $4.92^{\mathrm{a}}$ & $2.26^{b}$ & $2.88^{\mathrm{b}}$ & $3.19^{b}$ & $1.69^{b}$ \\
\hline methyl 3-methyl-2-butenoate & LRI,MS & 1157 & 3.79 & 0.85 & 2.84 & 2.61 & 2.86 & 1.75 \\
\hline methyl hexanoate & LRI,MS & 1178 & 1.29 & 1.01 & 2.05 & 1.51 & 1.02 & 1.29 \\
\hline ethyl hexanoate & S,LRI,MS & 1228 & 0.06 & 0.23 & 0.31 & 0.50 & 0.35 & 0.12 \\
\hline hexyl acetate & S,LRI,MS & 1265 & $21.75^{b}$ & $89.41^{\mathrm{a}}$ & $25.62^{b}$ & $11.18^{\mathrm{b}}$ & $91.10^{\mathrm{a}}$ & $13.11^{\mathrm{b}}$ \\
\hline (Z)-3-hexen-1-yl acetate $(\mathrm{mg} / \mathrm{kg})$ & S,LRI,MS & 1309 & $0.27^{b}$ & $0.62^{\mathrm{a}}$ & $0.20^{\mathrm{b}}$ & $0.05^{\mathrm{b}}$ & $0.26^{\mathrm{b}}$ & $0.09^{b}$ \\
\hline (Z)-2-hexen-1-yl acetate & LRI,MS & 1326 & $1.52^{b}$ & $4.02^{\mathrm{a}}$ & $1.44^{\mathrm{b}}$ & $0.91^{\mathrm{b}}$ & $1.75^{\mathrm{b}}$ & $0.91^{\mathrm{b}}$ \\
\hline (E)-3-hexenyl butanoate & LRI,MS & 1454 & $2.23 \mathrm{abc}$ & $0.88^{\mathrm{c}}$ & $2.34^{\mathrm{ab}}$ & $1.47 \mathrm{bc}$ & $2.94^{\mathrm{a}}$ & $1.71^{\mathrm{abc}}$ \\
\hline 3-hydroxy-2,4,4-trimethylpentyl 2-methylpropanoate & MS & 1854 & $0.37^{c}$ & $0.16^{\mathrm{c}}$ & $4.94^{\mathrm{a}}$ & $1.06^{\mathrm{bc}}$ & $0.95^{b c}$ & $1.54^{\mathrm{b}}$ \\
\hline methyl cinnamoylglycinate & MS & 1960 & $3.44^{\mathrm{b}}$ & $3.34^{\mathrm{b}}$ & $6.22^{\mathrm{a}}$ & $2.62^{c}$ & $2.25^{c}$ & $3.33^{\mathrm{b}}$ \\
\hline triacetin & LRI,MS & 2049 & $1.09 \mathrm{bc}$ & $0.41^{\mathrm{c}}$ & $5.19^{\mathrm{a}}$ & $1.44^{\mathrm{bc}}$ & $1.02 \mathrm{bc}$ & $1.77^{\mathrm{b}}$ \\
\hline 2-propenyl pentanoate & MS & 2074 & $3.07^{b c}$ & $1.38^{\mathrm{cd}}$ & $7.81^{a}$ & $3.79^{b}$ & $2.39 \mathrm{bcd}$ & $0.80^{\mathrm{d}}$ \\
\hline $\begin{array}{c}\text { methyl 3-oxo-2-pentyl-cyclopentaneacetate } \\
\text { benzenoids }\end{array}$ & MS & 2257 & $7.59^{a b}$ & $3.69^{c}$ & $12.29^{\mathrm{a}}$ & $8.44^{\mathrm{ab}}$ & $8.06^{\mathrm{ab}}$ & $6.03^{b c}$ \\
\hline benzene & LRI,MS & 926 & 9.83 & 14.67 & 14.44 & 12.05 & 11.62 & 10.10 \\
\hline toluene $(\mathrm{mg} / \mathrm{kg})$ & LRI,MS & 1027 & 0.10 & 0.17 & 0.07 & 0.40 & 0.17 & 0.10 \\
\hline$m$-xylene & LRI,MS & 1122 & 22.55 & 19.69 & 15.30 & 105.52 & 26.14 & 17.65 \\
\hline p-xylene $(\mathrm{mg} / \mathrm{kg})$ & LRI,MS & 1128 & 0.06 & 0.06 & 0.05 & 0.22 & 0.07 & 0.05 \\
\hline$o$-xylene $(\mathrm{mg} / \mathrm{kg})$ & LRI,MS & 1172 & 0.03 & 0.03 & 0.02 & 0.11 & 0.04 & 0.03 \\
\hline$p$-ethyltoluene & LRI,MS & 1213 & 16.73 & 17.37 & 11.75 & 56.70 & 14.44 & 11.29 \\
\hline 1,3,5-trimethylbenzene (mesitylene) & LRI,MS & 1235 & 11.77 & 11.52 & 12.45 & 32.07 & 9.69 & 8.84 \\
\hline 2-ethyltoluene & LRI,MS & 1251 & 7.00 & 6.28 & 5.39 & 18.11 & 5.10 & 4.07 \\
\hline p-cymene & S,LRI,MS & 1262 & 1.51 & 1.57 & 2.15 & 2.87 & 31.68 & 4.46 \\
\hline$m$-cymene & LRI,MS & 1270 & 19.46 & 16.99 & 16.47 & 40.75 & 10.97 & 10.45 \\
\hline 1,3-diethylbenzene & LRI,MS & 1293 & 1.54 & 0.75 & 0.84 & 2.14 & 0.59 & 0.71 \\
\hline
\end{tabular}


Table 1. Cont.

\begin{tabular}{|c|c|c|c|c|c|c|c|c|}
\hline \multirow{2}{*}{ Compound } & \multirow{2}{*}{ ID } & \multirow{2}{*}{ LRI } & \multicolumn{6}{|c|}{ Variety } \\
\hline & & & Buža & I. bjelica & Rosinjola & Oblica & Lastovka & Leccino \\
\hline$o$-cymene & LRI,MS & 1296 & 1.64 & 1.38 & 1.37 & 3.62 & 0.94 & 1.09 \\
\hline 1-ethyl-3,5-dimethylbenzene & LRI,MS & 1316 & 4.25 & 4.17 & 3.80 & 8.75 & 2.79 & 3.11 \\
\hline 1,2,3-trimethylbenzene (hemellitol) & LRI,MS & 1324 & 8.25 & 6.56 & 7.35 & 14.88 & 4.70 & 4.04 \\
\hline anisole & LRI,MS & 1329 & 3.77 & 2.45 & 2.91 & 1.27 & 3.42 & 1.85 \\
\hline 1,2,4,5-tetramethylbenzene & LRI,MS & 1413 & 1.89 & 1.36 & 1.44 & 3.99 & 1.13 & 1.09 \\
\hline isodurene & LRI,MS & 1422 & 2.78 & 2.21 & 2.22 & 5.44 & 2.06 & 1.80 \\
\hline 1,2,3,4-tetramethylbenzene & MS & 1423 & 2.78 & 2.20 & 2.20 & 5.39 & 2.01 & 1.81 \\
\hline p-cymenene & LRI,MS & 1436 & $9.05^{b}$ & $7.21^{\mathrm{c}}$ & $13.45^{\mathrm{a}}$ & $7.49^{c}$ & $6.48^{\mathrm{c}}$ & $6.87^{\mathrm{c}}$ \\
\hline methyl benzoate & LRI,MS & 1601 & $14.74^{\mathrm{b}}$ & $3.88^{c}$ & $22.08^{b}$ & $41.94^{\mathrm{a}}$ & $13.97^{b}$ & $4.01^{\mathrm{c}}$ \\
\hline acetophenone & S,LRI,MS & 1626 & $14.76^{\mathrm{b}}$ & $13.46^{\mathrm{b}}$ & $20.73^{a}$ & $13.08^{b}$ & $12.68^{b}$ & $13.60^{\mathrm{b}}$ \\
\hline estragole & LRI,MS & 1655 & $0.06^{c}$ & $0.03^{c}$ & $0.02^{\mathrm{c}}$ & $0.90^{\mathrm{b}}$ & $1.40^{\mathrm{a}}$ & $0.01^{\mathrm{c}}$ \\
\hline 4-ethylbenzaldehyde & LRI,MS & 1689 & $6.13^{b}$ & $4.73^{c}$ & $9.68^{a}$ & $4.66^{\mathrm{c}}$ & $3.77^{\mathrm{c}}$ & $4.66^{\mathrm{c}}$ \\
\hline methyl salicylate & LRI,MS & 1755 & $47.45^{\mathrm{ab}}$ & $18.17^{\mathrm{d}}$ & $62.13^{\mathrm{a}}$ & $61.68^{a}$ & $37.24 \mathrm{bc}$ & $30.00^{\mathrm{c}}$ \\
\hline aromatic aldehyde I (n.i.) & MS & 1810 & $9.56^{\mathrm{b}}$ & $9.57^{b}$ & $18.54^{\mathrm{a}}$ & $6.75^{c}$ & $5.56^{\mathrm{c}}$ & $9.18^{\mathrm{c}}$ \\
\hline aromatic aldehyde II (n.i.) & MS & 1839 & $9.12^{b}$ & $9.29^{b}$ & $18.39^{\mathrm{a}}$ & $6.46^{\mathrm{c}}$ & $5.50^{\mathrm{c}}$ & $8.81^{\mathrm{c}}$ \\
\hline benzyl alcohol & S,LRI,MS & 1851 & $20.21^{c}$ & $22.03^{c}$ & $24.09 \mathrm{bc}$ & $23.65 \mathrm{bc}$ & $35.74 \mathrm{ab}$ & $39.17^{\mathrm{a}}$ \\
\hline 2-phenylethyl alcohol (mg/kg) & S,LRI,MS & 1885 & 0.33 & 0.30 & 0.39 & 0.35 & 0.32 & 0.38 \\
\hline benzyl nitrile & LRI,MS & 1896 & $0.04^{\mathrm{b}}$ & $0.00^{\mathrm{b}}$ & $0.16^{\mathrm{b}}$ & $0.00^{\mathrm{b}}$ & $0.00^{\mathrm{b}}$ & $1.17^{\mathrm{a}}$ \\
\hline lilial & LRI,MS & 2020 & $1.55^{\mathrm{bc}}$ & $1.58^{b c}$ & $1.04^{b c}$ & $2.42^{\mathrm{ab}}$ & $3.74^{\mathrm{a}}$ & $1.01^{\mathrm{c}}$ \\
\hline methyl 2-methoxybenzoate & LRI,MS & 2037 & $5.21^{\mathrm{a}}$ & $0.43^{c}$ & $4.21^{\mathrm{ab}}$ & $3.49^{\mathrm{a} b}$ & $2.45^{b c}$ & $1.19^{\mathrm{c}}$ \\
\hline complex benzenoid (n.i.) & MS & 2041 & $3.94^{b}$ & $5.40^{\mathrm{b}}$ & $3.69^{b}$ & $12.24^{\mathrm{a}}$ & $11.25^{\mathrm{a}}$ & $3.07^{b}$ \\
\hline 2-phenoxyethanol & LRI,MS & 2106 & $3.30^{b c}$ & $2.07^{\mathrm{c}}$ & $4.99 \mathrm{bc}$ & $10.29^{a}$ & $7.11^{a b}$ & $5.49^{b c}$ \\
\hline methyl anthranilate & LRI,MS & 2200 & $0.73^{\mathrm{b}}$ & $0.05^{c}$ & $0.76^{\mathrm{b}}$ & $1.15^{\mathrm{b}}$ & $2.04^{\mathrm{a}}$ & $0.01^{\mathrm{c}}$ \\
\hline menthyl salicylate & MS & 2273 & $1.25^{\mathrm{b}}$ & $2.57^{\mathrm{a}}$ & $2.16^{\mathrm{ab}}$ & $2.74^{\mathrm{a}}$ & $2.27^{\mathrm{a}}$ & $1.39^{b}$ \\
\hline 4-ethoxystyrene & MS & 2348 & $1.97^{c}$ & $1.96^{\mathrm{c}}$ & $2.20^{b c}$ & $3.07^{\mathrm{a}}$ & $2.74^{a b}$ & $1.95^{\mathrm{c}}$ \\
\hline $\begin{array}{l}\text { benzoic acid } \\
\text { furanoids }\end{array}$ & LRI,MS & 2381 & $11.32^{b}$ & $44.78^{a}$ & $38.56^{\mathrm{a}}$ & $5.62^{b}$ & $7.75^{b}$ & $14.51^{\mathrm{b}}$ \\
\hline 2-ethylfuran $(\mathrm{mg} / \mathrm{kg})$ & LRI,MS & 938 & $0.14^{\mathrm{b}}$ & $0.08^{\mathrm{cd}}$ & $0.22^{\mathrm{a}}$ & $0.09 \mathrm{bc}$ & $0.05^{\mathrm{cd}}$ & $0.03^{\mathrm{d}}$ \\
\hline 2-vinylfuran & LRI,MS & 1059 & $10.26^{b}$ & $5.89 \mathrm{bc}$ & $17.85^{\mathrm{a}}$ & $9.15^{b}$ & $5.32 \mathrm{bc}$ & $1.94^{\mathrm{c}}$ \\
\hline 4-methyl-2,3-dihydrofuran & LRI,MS & 1184 & $4.98^{\mathrm{ab}}$ & $4.59^{b}$ & $3.83^{b}$ & $6.13^{\mathrm{a}}$ & $4.90^{\mathrm{ab}}$ & $3.86^{\mathrm{b}}$ \\
\hline 2-pentylfuran & LRI,MS & 1225 & 7.81 & 7.60 & 7.33 & 8.82 & 10.79 & 62.68 \\
\hline 5-methyl-2-furancarboxaldehyde & LRI,MS & 1551 & $4.99^{b}$ & $3.33^{c}$ & $7.19^{\mathrm{a}}$ & $3.58^{\mathrm{bc}}$ & $1.90^{\mathrm{cd}}$ & $1.55^{\mathrm{d}}$ \\
\hline $2(5 \mathrm{H})$-furanone & LRI,MS & 1722 & 5.90 & 3.67 & 5.23 & 2.23 & 1.42 & 2.94 \\
\hline
\end{tabular}


Table 1. Cont.

\begin{tabular}{|c|c|c|c|c|c|c|c|c|}
\hline \multirow{2}{*}{ Compound } & \multirow{2}{*}{ ID } & \multirow{2}{*}{ LRI } & \multicolumn{6}{|c|}{ Variety } \\
\hline & & & Buža & I. bjelica & Rosinjola & Oblica & Lastovka & Leccino \\
\hline 5-ethyl-2(5H)-furanone $(\mathrm{mg} / \mathrm{kg})$ & LRI,MS & 1733 & $0.19^{\mathrm{a}}$ & $0.08^{b c}$ & $0.19^{\mathrm{a}}$ & $0.15^{\mathrm{ab}}$ & $0.06^{\mathrm{bc}}$ & $0.03^{c}$ \\
\hline $\begin{array}{l}\text { 2-ethyl-5-methyl-tetrahydrofuran }(\mathrm{mg} / \mathrm{kg}) \\
\text { miscellaneous }\end{array}$ & MS & 1933 & $0.44^{\mathrm{ab}}$ & $0.21^{\mathrm{cd}}$ & $0.58^{\mathrm{a}}$ & $0.34 \mathrm{bc}$ & $0.15^{\mathrm{cd}}$ & $0.07^{\mathrm{d}}$ \\
\hline dimethyl sulfide & LRI,MS & 739 & $1.21 \mathrm{ab}$ & $1.26^{\mathrm{ab}}$ & $2.49^{\mathrm{a}}$ & $1.18^{\mathrm{ab}}$ & $0.74^{\mathrm{b}}$ & $1.81^{\mathrm{ab}}$ \\
\hline 2,4-dihydro-5-methyl-3H-pyrazol-3-one I & MS & 978 & 0.90 & 2.14 & 1.10 & 10.00 & 4.62 & 0.38 \\
\hline 1,2-dihydro-5-methyl-3H-pyrazol-3-one & MS & 1109 & 0.85 & 1.17 & 0.52 & 4.51 & 2.12 & 0.09 \\
\hline 2,4-dihydro-5-methyl-3H-pyrazol-3-one II & MS & 1150 & 0.08 & 0.35 & 0.09 & 1.82 & 0.60 & 0.01 \\
\hline 2-phenyl-1H-indole & MS & 1502 & $78.56^{\mathrm{c}}$ & $93.8^{\mathrm{b}}$ & $139.59^{\mathrm{a}}$ & $67.51^{\mathrm{c}}$ & $74.11^{\mathrm{c}}$ & $95.38^{\mathrm{b}}$ \\
\hline dimethyl sulfoxide & LRI,MS & 1540 & $26.39 \mathrm{abc}$ & $15.52^{\mathrm{c}}$ & $27.62^{a b}$ & $32.02^{\mathrm{a}}$ & $23.96^{\mathrm{abc}}$ & $16.65^{b c}$ \\
\hline n.i. $(m / z 189,207,131)$ & MS & 1688 & $0.51^{\mathrm{a}}$ & $0.21^{\mathrm{b}}$ & $0.45^{\mathrm{ab}}$ & $0.47^{\mathrm{a}}$ & $0.25^{\mathrm{ab}}$ & $0.19^{b}$ \\
\hline n.i. $(m / z 84,85,41,42,39,133,147,175)$ & MS & 1990 & $1.91^{\mathrm{b}}$ & $1.20^{b}$ & $3.72^{\mathrm{a}}$ & $1.07^{\mathrm{b}}$ & $1.32^{b}$ & $1.21^{\mathrm{b}}$ \\
\hline $\begin{array}{c}\text { phenol } \\
\text { totals }(m g / k g)\end{array}$ & S,LRI,MS & 1972 & $5.27^{c}$ & $6.01^{b}$ & $9.88^{\mathrm{a}}$ & $4.38^{\mathrm{d}}$ & $4.16^{\mathrm{d}}$ & $5.95^{b}$ \\
\hline total hydrocarbons & & & $2.12^{\mathrm{a}}$ & $1.54^{\mathrm{b}}$ & $1.99^{\mathrm{a}}$ & $1.13^{c}$ & $1.01^{\mathrm{c}}$ & $1.70^{\mathrm{b}}$ \\
\hline total monoterpenes & & & $0.35^{\mathrm{b}}$ & $0.23^{b}$ & $0.41^{b}$ & $0.71^{b}$ & $1.85^{\mathrm{a}}$ & $0.26^{b}$ \\
\hline total sesquiterpenes & & & $12.36^{\mathrm{a}}$ & $10.15^{a}$ & $9.29^{a}$ & $12.54^{\mathrm{a}}$ & $3.54^{b}$ & $2.66^{b}$ \\
\hline total aldehydes & & & $23.19^{b}$ & $24.78^{b}$ & $26.10^{b}$ & $14.79^{c}$ & $7.64^{c}$ & $36.37^{a}$ \\
\hline total ketones & & & $0.44^{\mathrm{b}}$ & $0.56^{\mathrm{a}}$ & $0.41^{b c}$ & $0.34^{c}$ & $0.33^{c}$ & $0.37^{c}$ \\
\hline total alcohols & & & $4.12^{\mathrm{bc}}$ & $5.02^{b c}$ & $4.95^{b c}$ & $5.69^{a b}$ & $7.89^{a}$ & $3.52^{c}$ \\
\hline total acids & & & $45.85^{b}$ & $447.25^{\mathrm{a}}$ & $60.81^{b}$ & $48.19^{b}$ & $65.37^{b}$ & $33.99^{b}$ \\
\hline total esters & & & $0.52^{b}$ & $1.29^{\mathrm{a}}$ & $0.56^{\mathrm{b}}$ & $0.25^{\mathrm{b}}$ & $0.61^{\mathrm{b}}$ & $0.27^{\mathrm{b}}$ \\
\hline total benzenoids & & & 0.80 & 0.81 & 0.87 & 1.60 & 0.88 & 0.79 \\
\hline total furanoids & & & 0.80 & 0.40 & 1.04 & 0.61 & 0.28 & 0.76 \\
\hline total miscellaneous & & & $0.12^{b}$ & $0.12^{b}$ & $0.19^{\mathrm{a}}$ & $0.12^{b}$ & $0.11^{b}$ & $0.12^{b}$ \\
\hline
\end{tabular}

ID-identification of compounds; S-retention time and mass spectrum consistent with that of the pure standard and with NIST05 mass spectra electronic library; LRI-linear retention index consistent with that found in literature; MS-mass spectra consistent with that from NIST05 mass spectra electronic library or literature; n.i.-not identified. The compounds with only MS symbol in ID column were tentatively identified. The compounds for which pure standards were not available (without symbol S in the ID column) were quantified semi-quantitatively and their concentrations were expressed as equivalents of compounds with similar chemical structure assuming a response factor $=1$. Different superscript lowercase letters in a row represent statistically significant differences between mean values at $p<0.05$ obtained by one-way ANOVA and least significant difference (LSD) test. 


\subsubsection{Ketones}

Istrian EVOOs contained higher concentration of the most important olive oil ketone in sensory terms, 1-penten-3-one, in relation to the Dalmatian ones, especially Lastovka (Table 1). The highest concentrations of 2-cyclohehene-1,4-dione were found in Rosinjola followed by Buža, while the lowest were found in Leccino EVOO. Dalmatian Oblica and Lastovka EVOOs were distinguished by the highest concentration of 1-(2,6,6-trimethyl-1-cyclohexene-1-yl)-1-penten-3-one and the lowest concentration of $(Z)$-cinerolone. The latter volatile compound was found to be a marker of Leccino, the same as 4'-ethoxy-2'-hydroxyoctadecanophenone was for Rosinjola EVOO.

\subsubsection{Alcohols}

Among LOX-generated unsaturated C6 alcohols, a similar pattern as in the case of 3-hexenals was observed, with Leccino EVOO containing the lowest concentration of both (E)- and (Z)-3-hexen-1-ol (Table 1). Dalmatian EVOOs, especially Lastovka in the case of (E)-2-hexen-1-ol, exhibited the highest concentrations. 1-Penten-3-ol turned out to be a marker of I. bjelica EVOO, Rosinjola EVOO was the most abundant in 2-ethyl-1-hexanol and 2-(2-butoxyethoxy)-ethanol, while higher concentration of a number of non-LOX alcohols, such as 1-methoxy-2-propanol, 3-methyl-1-butanol, and 1-pentanol, turned out to be a feature of Lastovka EVOO.

\subsubsection{Acids}

Leccino EVOO was characterized by the lowest concentration of (E)-3-hexenoic acid (Table 1). Dalmatian, especially Lastovka EVOO, had the highest concentrations of butanoic and hexanoic acids, while I. bjelica EVOO was by far the most abundant in other middle-chain volatile fatty acids, especially nonanoic acid, as well as total acids. Rosinjola was distinguished by higher levels of 2-ethylhexanoic acid, which corresponded well to the higher concentration of 2-ethyl-1-hexanol found in this EVOO.

\subsubsection{Esters}

Istarska bjelica EVOO exhibited the highest concentration of the acetates of C6 alcohols, methyl acetate, and total esters (Table 1). Lastovka EVOO was abundant in hexyl acetate. Rosinjola EVOO stood out with the highest levels of several, mostly tentatively identified esters with high LRIs.

\subsubsection{Benzenoids}

For many simple benzenoids (e.g., xylenes, ethylbenzenes, cymenes, etc.) no statistically significant differences between the monovarietal EVOOs were observed (Table 1). Rosinjola was the most distinguished by the highest concentrations of $p$-cymenene, acetophenone, 4-ethylbenzaldehyde, and the two non-identified aromatic aldehydes. Estragole and methyl anthranilate were found in the highest concentration in Lastovka EVOO, and these two compounds, together with lilial, 2-phenoxyethanol, and 4-ethoxystyrene, were more abundant in Dalmatian than in Istrian EVOOs. The highest concentration of benzyl nitrile was observed in Leccino EVOO. Low concentrations of several benzenoids were characteristic for particular EVOOs: methyl salicylate in I. bjelica, and methyl 2-methoxybenzoate and methyl anthranilate in I. bjelica and Leccino.

\subsubsection{Furanoids}

Similar as for the benzenoids, Rosinjola EVOO was characterized by several furanoid markers, including 2-ethylfuran, 2-vinylfuran, and 5-methyl-2-furancarboxaldehyde (Table 1). On the other hand, Leccino EVOO had the lowest concentration of furanoids in general.

\subsubsection{Miscellaneous Compounds}

Rosinjola EVOO contained the highest concentration of 2-phenyl-1H-indole and phenol (Table 1). Phenol concentration was the lowest in Dalmatian Oblica and Lastovka EVOOs. 


\subsubsection{Odor Activity Values (OAV)}

Table 2 lists the average odor activity values (OAV) of the volatile aroma compounds found in the investigated EVOOs, calculated as the ratios of their concentrations and odor perception thresholds available in literature. For thirteen compounds average OAV higher than 1 was observed in at least one of the monovarietal EVOOs implying their direct influence on the aroma. The compound with the highest $\mathrm{OAV}$ was (Z)-3-hexenal, followed by 1-penten-3-one and (E)-2-hexenal, while other compounds exhibited much lower OAVs. (Z)-3-hexenal was potentially the most important odorant in all the investigated monovarietal EVOOs except I. bjelica and Leccino in which 1-penten-3-one was dominant.

Table 2. Sensory descriptors and odor perception thresholds of volatile aroma compounds in monovarietal extra virgin olive oils produced from Buža, Istarska bjelica, Rosinjola, Oblica, Lastovka, and Leccino varieties in Croatia, sorted in descending order according to their average odor activity values $(\mathrm{OAV})$.

\begin{tabular}{|c|c|c|c|c|c|c|c|c|}
\hline \multirow{2}{*}{ Volatile Compound } & \multirow{2}{*}{$\begin{array}{l}\text { Sensory Descriptor } \\
\text { (Aroma)* }\end{array}$} & \multirow{2}{*}{ Threshold * } & \multicolumn{6}{|c|}{ Odor Activity Value (OAV) } \\
\hline & & & Buža & I. bjelica & Rosinjola & Oblica & Lastovka & Leccino \\
\hline \multicolumn{9}{|l|}{$O A V>1$} \\
\hline (Z)-3-hexenal & leaf-like, green, apple-like & 1.7 & $800.00^{a}$ & $235.29 \mathrm{~cd}$ & $564.71^{\mathrm{abc}}$ & $641.18^{a b}$ & $\begin{array}{c}276.47 \\
\text { bcd }\end{array}$ & $82.35^{\mathrm{d}}$ \\
\hline 1-penten-3-one & leaf, green, pungent, sweet & 0.73 & $397.26^{a}$ & $397.26^{a}$ & $356.16^{\mathrm{ab}}$ & $246.58^{b c}$ & $164.38^{c}$ & $301.37^{a b}$ \\
\hline (E)-2-hexenal & $\begin{array}{c}\text { green, apple-like, bitter } \\
\text { almond }\end{array}$ & 420 & $46.14^{b c}$ & $53.67^{\mathrm{b}}$ & $52.29^{b}$ & $28.33^{\mathrm{cd}}$ & $14.10^{\mathrm{d}}$ & $83.21^{\mathrm{a}}$ \\
\hline hexanal & $\begin{array}{c}\text { green, sweet, green apple, } \\
\text { grassy }\end{array}$ & 75 & $3.20^{\mathrm{b}}$ & $3.87^{b}$ & $3.60^{b}$ & $5.87^{\mathrm{a}}$ & $7.07^{\mathrm{a}}$ & $2.67^{b}$ \\
\hline 3-methylbutanal & malty & 5.2 & $2.02^{b}$ & $4.29^{\mathrm{a}}$ & $2.05^{b}$ & $1.63^{\mathrm{b}}$ & $2.37^{b}$ & $2.36^{\mathrm{b}}$ \\
\hline 1-hexanol & fruit, banana, soft, grass & 400 & 1.75 & 3.03 & 3.00 & 2.93 & 3.95 & 1.90 \\
\hline 2-methylbutanal & malty & 5.4 & 1.99 & 2.48 & 2.21 & 1.46 & 3.46 & 2.55 \\
\hline hexanoic acid & pungent, rancid, sweaty & 700 & $1.09^{\mathrm{c}}$ & $1.76^{\mathrm{bc}}$ & $1.89^{b c}$ & $2.49^{\mathrm{ab}}$ & $3.16^{\mathrm{a}}$ & $1.43^{c}$ \\
\hline $\begin{array}{l}\text { (Z)-3-hexen-1-yl } \\
\text { acetate }\end{array}$ & $\begin{array}{l}\text { green, banana-like, olive } \\
\text { fruity }\end{array}$ & 200 & $1.35^{b}$ & $3.10^{\mathrm{a}}$ & $1.00^{b}$ & $0.25^{b}$ & $1.30^{b}$ & $0.45^{b}$ \\
\hline (E)-2-octenal & herbaceous, spicy & 4 & $2.94^{\mathrm{a}}$ & $1.38^{\mathrm{c}}$ & $2.03^{b c}$ & $2.62^{\mathrm{ab}}$ & $1.79 \mathrm{bc}$ & $2.29^{b}$ \\
\hline octanoic acid & oily, fatty & 3000 & $0.16^{\mathrm{b}}$ & $2.61^{\mathrm{a}}$ & $0.43^{\mathrm{b}}$ & $0.55^{\mathrm{b}}$ & $0.49^{b}$ & $0.20^{b}$ \\
\hline (Z)-3-hexen-1-ol & green, apple, leaf-like, banana & 1100 & $1.48^{\mathrm{b}}$ & $0.98^{b c}$ & $1.36^{\mathrm{bc}}$ & $2.52^{\mathrm{a}}$ & $1.75^{\mathrm{ab}}$ & $0.55^{c}$ \\
\hline $\begin{array}{c}\text { ethyl } \\
\text { 2-methylbutanoate } \\
O A V<1\end{array}$ & fruity & 0.72 & 1.25 & 0.89 & 0.76 & 0.97 & 0.97 & 0.71 \\
\hline 1-penten-3-ol & lawn, olive, leaf, pungent & 400 & $0.45^{\mathrm{b}}$ & $0.70^{\mathrm{a}}$ & $0.48^{\mathrm{b}}$ & $0.35^{\mathrm{b}}$ & $0.48^{\mathrm{b}}$ & $0.45^{\mathrm{b}}$ \\
\hline (E)-2-hexen-1-ol & green, grass, leaves, sweet & 5000 & $0.20^{\mathrm{b}}$ & $0.34^{\mathrm{b}}$ & $0.27^{b}$ & $0.21^{\mathrm{b}}$ & $0.68^{a}$ & $0.28^{b}$ \\
\hline 3-methyl-1-butanol & woody, whiskey, sweet & 100 & $0.20^{b}$ & $0.27^{\mathrm{b}}$ & $0.23^{b}$ & $0.27^{\mathrm{b}}$ & $0.58^{a}$ & $0.24^{b}$ \\
\hline octanal & fatty, sharp, citrus-like, soapy & 320 & $0.31^{\mathrm{a}}$ & $0.34^{\mathrm{a}}$ & $0.28^{a}$ & $0.22^{b}$ & $0.22^{b}$ & $0.28^{\mathrm{a}}$ \\
\hline (E)-2-penten-1-ol & green fruity, fresh olive fruits & 250 & 0.25 & 0.26 & 0.26 & 0.18 & 0.27 & 0.24 \\
\hline (E)-2-pentenal & green, apple, bitter almond & 300 & $0.17^{\mathrm{a}}$ & $0.16^{\mathrm{a}}$ & $0.15^{\mathrm{ab}}$ & $0.13^{\mathrm{ab}}$ & $0.08^{\mathrm{b}}$ & $0.14^{\mathrm{ab}}$ \\
\hline butanoic acid & rancid, cheese & 650 & $0.04^{c}$ & $0.06^{\mathrm{bc}}$ & $0.06^{\mathrm{bc}}$ & $0.08^{a b}$ & $0.11^{a}$ & $0.05^{\mathrm{bc}}$ \\
\hline$(E, Z)-2,4$-decadienal & deep-fried & 10 & $0.07^{\mathrm{b}}$ & $0.03^{c}$ & $0.05^{b c}$ & $0.11^{\mathrm{a}}$ & $0.09^{a b}$ & $0.06^{\mathrm{bc}}$ \\
\hline hexyl acetate & green, fruity, sweet, apple & 1040 & $0.02^{b}$ & $0.09^{a}$ & $0.02^{b}$ & $0.01^{b}$ & $0.09^{a}$ & $0.01^{\mathrm{b}}$ \\
\hline 1-pentanol & fruity, strong, sticky, balsamic & 470 & $0.02^{b c}$ & $0.03 \mathrm{ab}$ & $0.02 \mathrm{bc}$ & $0.04^{\mathrm{a}}$ & $0.05^{\mathrm{a}}$ & $0.01^{\mathrm{c}}$ \\
\hline octane & sweety, alcane & 940 & 0.03 & 0.04 & 0.03 & 0.03 & 0.04 & 0.03 \\
\hline 2-octanone & mould, green & 510 & 0.01 & 0.00 & 0.00 & 0.00 & 0.00 & 0.00 \\
\hline (E)-3-hexen-1-ol & green, bitter & 1500 & $0.02^{a b}$ & $0.02^{a b}$ & $0.02^{\mathrm{ab}}$ & $0.03^{\mathrm{a}}$ & $0.03^{\mathrm{a}}$ & $0.01^{b}$ \\
\hline 1-nonanol & fatty, rancid & 280 & 0.01 & 0.01 & 0.01 & 0.01 & 0.01 & 0.01 \\
\hline heptanal & oily, fatty, woody & 500 & 0.01 & 0.01 & 0.01 & 0.01 & 0.01 & 0.01 \\
\hline$(E, E)$-2,4-heptadienal & fatty, rancid & 3620 & $0.01^{b}$ & $0.01^{\mathrm{d}}$ & $0.01 \mathrm{bcd}$ & $0.01 \mathrm{bc}$ & $0.01^{\mathrm{c}}$ & $0.01^{\mathrm{a}}$ \\
\hline$(E, E)$-2,4-decadienal & deep-fried & 180 & $0.00^{b c}$ & $0.00^{c}$ & $0.01^{\mathrm{ab}}$ & $0.01^{\mathrm{a}}$ & $0.00^{b}$ & $0.00^{b}$ \\
\hline 6-methyl-5-hepten-2-one & pungent, green & 1000 & $0.00^{b c}$ & $0.00^{b c}$ & $0.00^{\mathrm{abc}}$ & $0.00^{\mathrm{ab}}$ & $0.00^{\mathrm{a}}$ & $0.00^{c}$ \\
\hline 3-pentanone & fruity, green, sweet & 70,000 & $0.00^{c}$ & $0.00^{\mathrm{a}}$ & $0.00^{c}$ & $0.00^{b c}$ & $0.00^{\mathrm{ab}}$ & $0.00^{c}$ \\
\hline
\end{tabular}

* sensory descriptors and odor perception thresholds $(\mu \mathrm{g} / \mathrm{kg}$ oil) reported in literature [21,44-47]. Values in bold indicate the highest average OAV for a given volatile compound among monovarietal extra virgin olive oils.

\subsubsection{Multivariate Statistical Analysis}

A differentiation model built by SLDA classified correctly all the monovarietal EVOOs according to variety (Figure 1) and extracted 30 variables (Table S2). A 100\% correct classification was obtained after including 22 variables. Phenol was included in the model as the first and classified correctly $41.76 \%$ of all the investigated EVOO samples. After subsequently including nonanoic acid, $\alpha$-muurolene, 3,7-decadiene I, and estragole (five compounds in total), the total percentage of the correctly classified EVOOs increased to $94.51 \%$. 


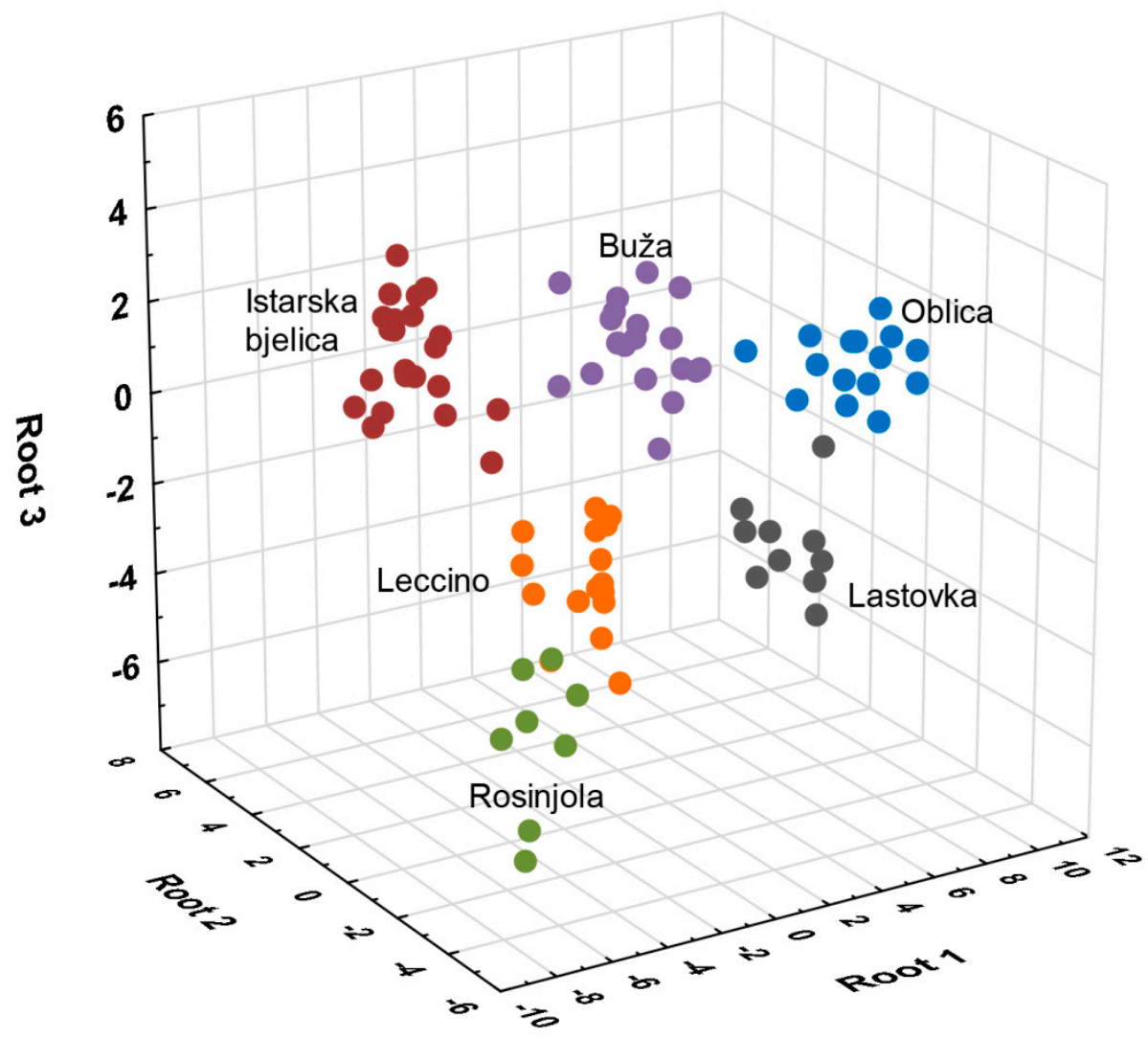

Figure 1. Separation of monovarietal extra virgin olive oils produced from Buža, Istarska bjelica, Rosinjola, Oblica, Lastovka, and Leccino varieties in Croatia according to variety in three-dimensional space defined by the first three discriminant functions (roots) on the basis of volatile aroma compound composition.

Unsaturated hydrocarbons from the LOX pathway, accompanied by the most important LOX volatile (Z)-3-hexenal, were characterized by the highest positive VIP scores obtained by PLSDA and were confirmed to be typical for Buža EVOO (Figure 2a). Middle-chain fatty acids and hexenol acetates were the volatiles with the highest VIP scores in I. bjelica EVOO (Figure 2b), while the markers of Rosinjola EVOO were mostly benzenoids (Figure 2c). The volatile compound with by far the highest VIP score for the discrimination of Oblica was methyl benzene (Figure 2d), while Lastovka EVOO typicity was mostly owed to its abundance in terpenes and deficiency in LOX volatiles (Figure 2e). High concentrations of benzyl nitrile, $(E)$-2-hexenal, and $(E, E)$-2,4-heptadienal, as well as low concentrations of sesquiterpenes, were confirmed to be the most prominent typical characteristics of the Leccino EVOO volatile profile (Figure 2f). 

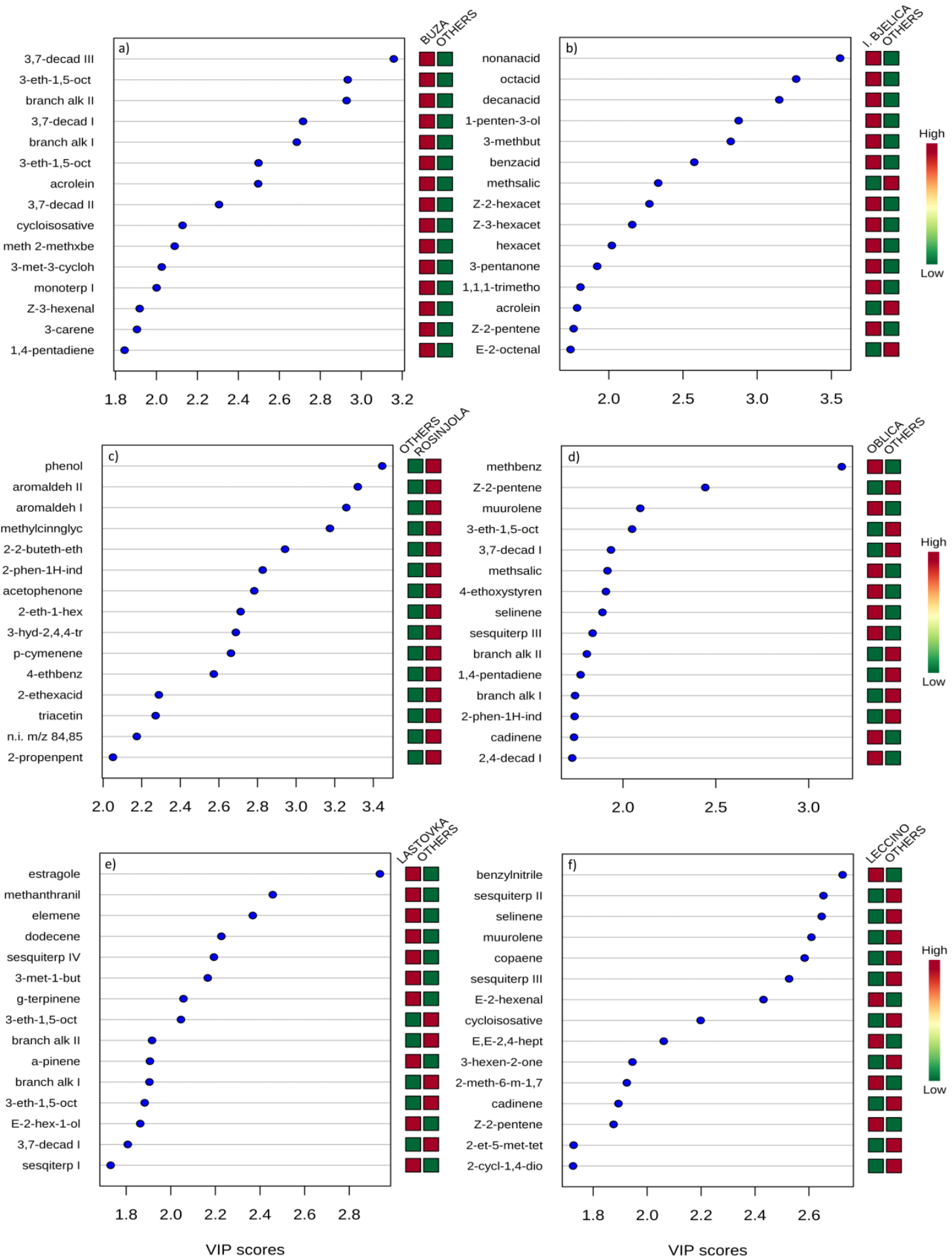

Figure 2. Variable Importance in Projection (VIP) scores of the variables (volatile compounds) most useful for the differentiation of each monovarietal extra virgin olive oil (EVOO), specifically: (a) Buža, (b) Istarska bjelica, (c) Rosinjola, (d) Oblica, (e) Lastovka, and (f) Leccino from the other five EVOOs produced in Croatia. Variables were extracted by partial least squares discriminant analysis applied on mean-centered data of six separate datasets each including two groups (a single vs. other five monovarietal EVOO). 


\subsection{Phenols}

Nineteen phenolic compounds were identified in total, including simple phenols, phenolic acids, flavonoids, lignans, and secoiridoids (Table 3). For many of those significant differences between average concentrations in the investigated EVOOs were found, and a few phenols emerged as exclusive markers of particular monovarietal EVOOs. Lastovka EVOO was generally characterized by the highest concentrations of simple phenols (except vanillin) and $p$-coumaric acid. Leccino turned out to be clearly distinguishable from the other monovarietal EVOOs by the highest concentration of vanillin, and the lowest concentrations of $p$-coumaric acid and luteolin. Among lignans, high pinoresinol content was characteristic for Buža EVOO. The secoiridoid profiles differed among the investigated monovarietal EVOOs. The concentration of one of the major oleuropein aglycons and phenols in general in olive oil, dialdehydic form of decarboxymethylelenolic acid linked to hydroxytyrosol, i.e., 3,4-DHPEA-EDA or oleacein, was the highest in Leccino EVOO. Its tyrosol-based analogue, the major aglycon of ligstroside, $p$-HPEA-EDA or oleocanthal, clearly distinguished two groups of EVOO, I. bjelica, Oblica, and Leccino with higher, and Buža, Rosinjola, and Lastovka EVOOs with lower concentrations. The composition of other oleuropein and ligstroside aglycons also turned out to be variety-specific; it is worth mentioning low concentration of oleuropein aglycon I in Oblica and Leccino, low concentration of oleuropein aglycon II in Oblica, and exceptionally higher concentration of oleuropein + ligstroside aglycons in I. bjelica than in other EVOOs.

Table 3. Concentrations $(\mathrm{mg} / \mathrm{kg})$ of phenols determined by ultra-performance liquid chromatography with diode-array detection (UPLC-DAD) in monovarietal extra virgin olive oils produced from Buža, Istarska bjelica, Rosinjola, Oblica, Lastovka, and Leccino varieties in Croatia.

\begin{tabular}{|c|c|c|c|c|c|c|}
\hline \multirow{2}{*}{ Phenol } & \multicolumn{6}{|c|}{ Variety } \\
\hline & Buža & I. bjelica & Rosinjola & Oblica & Lastovka & Leccino \\
\hline \multicolumn{7}{|l|}{ simple phenols } \\
\hline tyrosol & $4.87^{b}$ & $11.29^{\mathrm{a}}$ & $3.69^{b}$ & $9.10^{\mathrm{ab}}$ & $12.28^{a}$ & $5.60^{b}$ \\
\hline hydroxytyrosol & $5.40^{\mathrm{c}}$ & $10.21^{b}$ & $5.59 \mathrm{bc}$ & $6.33^{b c}$ & $20.17^{\mathrm{a}}$ & $6.47^{b c}$ \\
\hline hydroxytyrosol acetate * & $0.35^{\mathrm{c}}$ & $0.67^{\mathrm{b}}$ & $0.37^{b c}$ & $0.42^{b c}$ & $1.44^{\mathrm{a}}$ & $0.50^{b c}$ \\
\hline vanillin & $0.21^{\mathrm{b}}$ & $0.16^{b c}$ & $0.20^{b c}$ & $0.12^{c}$ & $0.11^{\mathrm{c}}$ & $0.31^{\mathrm{a}}$ \\
\hline \multicolumn{7}{|l|}{ phenolic acids } \\
\hline vanillic acid & 0.31 & 0.32 & 0.33 & 0.18 & 0.37 & 0.25 \\
\hline $\begin{array}{l}p \text {-coumaric acid } \\
\text { flavonoids }\end{array}$ & $1.26^{\mathrm{bc}}$ & $0.90^{\mathrm{c}}$ & $0.82^{\mathrm{cd}}$ & $1.69^{\mathrm{b}}$ & $2.80^{a}$ & $0.34^{\mathrm{d}}$ \\
\hline luteolin & $2.02^{b c}$ & $2.95^{\mathrm{a}}$ & $2.86^{\mathrm{ab}}$ & $2.93^{\mathrm{a}}$ & $3.35^{\mathrm{a}}$ & $1.89^{\mathrm{c}}$ \\
\hline apigenin & $0.55^{b c}$ & $0.87^{\mathrm{a}}$ & $0.66^{b}$ & $0.33^{\mathrm{d}}$ & $0.39^{\mathrm{d}}$ & $0.46^{\mathrm{cd}}$ \\
\hline \multicolumn{7}{|l|}{ lignans } \\
\hline pinoresinol & $9.97^{\mathrm{a}}$ & $4.02^{\mathrm{c}}$ & $6.98^{b}$ & $3.21^{\mathrm{c}}$ & $3.68^{c}$ & $4.14^{\mathrm{c}}$ \\
\hline $\begin{array}{l}\text { Acetoxypinoresinol * } \\
\text { secoiridoids }\end{array}$ & $6.72^{\mathrm{c}}$ & $14.11^{\mathrm{a}}$ & $11.39 \mathrm{ab}$ & $8.69^{b c}$ & $11.94^{\mathrm{ab}}$ & $7.49^{\mathrm{c}}$ \\
\hline Secologanoside * & $0.03^{b}$ & $0.04^{\mathrm{b}}$ & $0.03^{b}$ & $0.04^{\mathrm{b}}$ & $0.06^{\mathrm{a}}$ & $0.04^{\mathrm{b}}$ \\
\hline elenolic acid glucoside * & $0.04^{b c}$ & $0.04^{\mathrm{c}}$ & $0.05^{\mathrm{abc}}$ & $0.05^{\mathrm{ab}}$ & $0.06^{\mathrm{a}}$ & $0.04^{\mathrm{c}}$ \\
\hline 3,4-DHPEA-EDA * & $95.50^{b}$ & $115.68^{b}$ & $104.93^{b}$ & $98.46^{b}$ & $121.33^{b}$ & $175.06^{\mathrm{a}}$ \\
\hline oleuropein aglycone I* & $72.56^{\mathrm{bc}}$ & $94.57^{\mathrm{ab}}$ & $109.85^{\mathrm{a}}$ & $49.09 \mathrm{~cd}$ & $115.05^{\mathrm{a}}$ & $41.17^{\mathrm{d}}$ \\
\hline$p$-HPEA-EDA * & $49.15^{b}$ & $82.70^{\mathrm{a}}$ & $47.35^{b}$ & $76.79^{\mathrm{a}}$ & $49.21^{b}$ & $87.49^{\mathrm{a}}$ \\
\hline oleuropein + ligstroside aglycones I \& II * & $43.38^{b}$ & $97.82^{\mathrm{a}}$ & $49.19^{b}$ & $38.63^{b}$ & $49.33^{b}$ & $30.11^{b}$ \\
\hline oleuropein aglycone II * & $64.44^{\mathrm{c}}$ & $79.72 \mathrm{abc}$ & $100.61^{\mathrm{a}}$ & $42.38^{\mathrm{d}}$ & $94.14^{\mathrm{ab}}$ & $71.77^{b c}$ \\
\hline ligstroside aglycon III * & $1.66^{\mathrm{c}}$ & $4.60^{\mathrm{a}}$ & $1.82^{\mathrm{c}}$ & $2.79 \mathrm{bc}$ & $1.99^{\mathrm{c}}$ & $4.04^{\mathrm{ab}}$ \\
\hline oleuropein aglycone III * & $9.06^{\mathrm{c}}$ & $15.84^{\mathrm{a}}$ & $11.66^{\mathrm{bc}}$ & $11.40^{\mathrm{bc}}$ & $13.75^{a b}$ & $9.85^{c}$ \\
\hline total phenols & $367.25^{c}$ & $536.49^{a}$ & $458.38^{a b c}$ & $352.63^{c}$ & $501.45^{a b}$ & $447.00^{b c}$ \\
\hline
\end{tabular}

* The phenols for which pure standards were not available were quantified semi-quantitatively and their concentrations were expressed as equivalents of phenols with similar chemical structure assuming a response factor $=1$. Different superscript lowercase letters in a row represent statistically significant differences between mean values at $p<0.05$ obtained by one-way ANOVA and least significant difference (LSD) test. 


\subsection{Sensory Attributes}

The majority of the investigated monovarietal EVOOs were characterized by common EVOO sensory attributes (Figure 3, Table S3). Buža EVOO showed higher intensities of the majority of the assessed positive odor attributes, and it was clearly distinguished from the others by the highest intensity of chicory/rocket. Istarska bjelica and Rosinjola had the lowest intensity of almond. The specificity of the odor of Oblica EVOO was contained mainly in the most intense green banana nuance, while Lastovka was distinguished as the only monovarietal EVOO with the woody note. Istrian EVOOs were generally described by higher intensities of green grass/leaves, aromatic herbs, and chicory/rocket attributes (with the exception of Leccino EVOO) in relation to the Dalmatian ones.
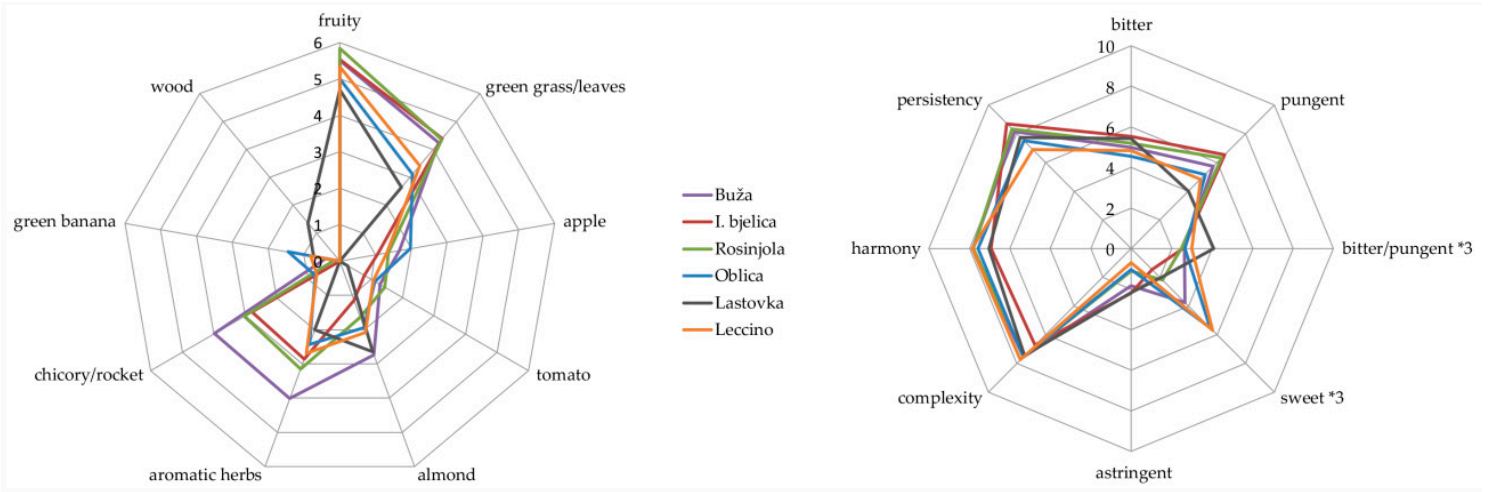

Figure 3. The intensities of the sensory attributes obtained by quantitative descriptive sensory analysis of monovarietal extra virgin olive oils (EVOOs) produced from Buža, Istarska bjelica, Rosinjola, Oblica, Lastovka, and Leccino varieties in Croatia $(* 3$ - the intensities of particular sensory attributes were multiplied by 3 to better visualize the differences between monovarietal EVOOs).

As regards the main EVOO taste attributes, bitterness and pungency, the EVOOs from Istrian native varieties Buža, I. bjelica, and Rosinjola generally showed higher intensities. The exception was the intensity of bitterness in Lastovka which was among the highest, which resulted in the highest bitterness/pungency ratio in this EVOO (Figure 3, Table S3). Oblica and Leccino were described as the sweetest among the EVOOs.

Istarska bjelica EVOO was characterized as the least complex, while I. bjelica and Lastovka EVOOs were less harmonious with respect to others. EVOOs from Istrian native varieties were the most, and Leccino the least persistent in terms of pungency.

\section{Discussion}

From the results of the GC-IT-MS and UPLC-DAD analysis of volatile compounds and phenols, respectively, it was clear that each of the investigated monovarietal EVOOs was characterized by a unique volatile and phenol profile. Since the samples were collected from various producers and were relatively heterogeneous in terms of geographical microlocations, growing conditions, harvest date, olive processing technology, and EVOO finalization and storage parameters, it could be assumed, with a high degree of certainty, that the effects of all of these factors were random, and that varietal origin was the main source of the observed differences. In fact, varietal origin was previously found to have a greater impact on volatile composition than various environmental factors [28]. However, the geographical origin possibly had an effect, which was impossible to evaluate separately from the effect of variety considering the varieties studied were specific for their regions. Istrian EVOOs, mostly those made of native Buža and Rosinjola, as well as those of the international variety Leccino, were characterized by higher concentrations of many LOX volatiles in relation to Dalmatian Oblica and Lastovka EVOO, including the most odoriferous ones, such as hexenals and 1-penten-3-one (Table 1). It is probable that this was directly reflected on the differences in their sensory profiles, since Istrian EVOOs had higher intensities of the majority of positive odor attributes, especially those 
of green grass/leaves and chicory/rocket (Figure 3, Table S3). Lower OAV values of the most potent odorants, (Z)-3-hexenal, 1-penten-3-one, and (E)-2-hexenal found in the Dalmatian, especially Lastovka EVOO, corroborated this assumption (Table 2). Since Istria is a region characterized by lower average temperatures than Dalmatia (Table S1), these results basically corroborated what was previously found in the majority of such studies that the temperature of environment is negatively correlated with the concentrations of LOX-derived volatile aroma compounds and the resulting EVOO positive sensory attributes [48,49].

Although without statistical significance in some cases, Buža EVOO excelled with the highest concentrations of the majority of positive LOX volatiles (Table 1), as well as with the highest intensities of positive odor sensory attributes (Figure 3, Table S3), which were probably in a causal relationship. It is worth emphasizing the highest cumulative odor activity value (OAV) of (Z)-3-hexenal and 1-penten-3-one, the two most powerful known odorants in EVOO with very low odor perception thresholds of 0.0017 and $0.00073 \mathrm{mg} / \mathrm{kg}$, respectively [22,46] (Table 2), which certainly exhibited key roles. A large proportion of LOX volatiles among those extracted by the PLSDA as the most significant VIP compounds discriminating Buža from the other EVOOs (Figure 2) corroborated the assumption that this variety is characterized by strong lipoxygenase and hydroperoxide lyase activities in the LOX pathway.

Generally, the most similar to Buža in terms of high concentrations of LOX volatiles (Table 1) and their OAVs (Table 2), as well as high intensities of positive odor attributes (Figure 3, Table S3), was Rosinjola EVOO. When it came down to the discriminating VIP compounds, those extracted by PLSDA were mostly benzenoids (Figure 2). Many benzenoids which were found in relatively high concentration in Rosinjola EVOO, including methyl benzoate, acetophenone, and methyl salicylate, were previously reported to be important almond odorants [50], however their impact in olive oil has not been investigated yet. As well, almond note was not especially accentuated in Rosinjola EVOO (Figure 3, Table S3).

Istarska bjelica had lower concentrations of many important LOX volatiles (Table 1). Since it is a late ripening variety [51] it is possible that it was characterized by a slightly weaker LOX enzymatic load with respect to Buža and Rosinjola EVOO. As it is known that phenols may act as LOX enzymatic activity inhibitors [52], the possibility that the high concentrations of phenols found in this monovarietal EVOO (Table 3) acted in this way during milling and malaxation should not be excluded. However, the concentrations and OAVs of some other major LOX odorants, such as $(E)$-2-hexenal and 1-penten-3-one, were relatively high, suggesting a notable activity of $(Z)-3:(E)-2$-enal isomerase which catalyzes the conversion of (Z)-3- to (E)-2-hexenal, as well as relatively high activity of the enzymes or availability of the substrates involved in the synthesis of $C 5$ compounds via 13-alkoxy radicals in this side-branch of the LOX pathway. The concentrations and OAVs of these volatiles were not lower that those found in Buža and Rosinjola EVOOs (Tables 1 and 2), so it is probable that (E)-2-hexenal and 1-penten-3-one were the key odorants in the formation of I. bjelica aroma and were the most responsible for the high intensity of several positive sensory attributes observed in this EVOO (Figure 3, Table S3). The most typical VIP chemical markers distinguishing I. bjelica EVOO were mostly non-LOX volatiles, namely middle-chain fatty acids and C6 alcohol acetates (Figure 2). Judging on the determined OAV values (Table 2), their sensory relevance was probably minor to medium. High concentrations of C6 alcohol acetates (Table 1) implied a possible high alcohol acyl transferase activity in olives and olive paste of this variety [53].

As stated previously, Oblica was characterized by a slightly lower contribution of the LOX volatiles and, consequently, lower intensities of particular positive odor attributes with respect to Istrian EVOOs, but was still superior to Lastovka EVOO (Table 1, Figure 3, Table S3). It was possibly mostly due to lower 1-penten-3-one and (E)-2-hexenal concentrations and OAVs, since the level of (Z)-3-hexenal was relatively high (Tables 1 and 2). As well, it is possible that a part of the fruity and green aroma originated from hexanal, found in higher concentration with respect to the Istrian EVOOs (Tables 1 and 2). Green banana odor sensory attribute which was found to be typical for Oblica EVOO (Figure 3, Table S3) could 
have, at least partly, originated from the volatiles often associated with this nuance. (Z)-3-hexen-1-ol was certainly a candidate for this role [45], since its concentration was the highest in this EVOO (Table 1) and at the same time above the corresponding odor detection threshold (Table 2). For other LOX volatiles with the odor commonly described as banana-like, such as hexanol, hexenyl acetates and penten-1-ols [45,54], no significant differences between varieties were found. As well, their levels in Oblica were not among the highest among the investigated EVOOs (Table 1), implying their impact in the formation of green banana nuance was probably not crucial. The same applies for other minor volatiles commonly reported as carriers of banana odor, such as isoamyl and other acetates. The VIP compounds responsible for the differentiation of Oblica EVOO (Figure 2), which pertained to several chemical families, could have not been meaningfully related to the occurrence of green banana odor.

Lastovka EVOO was characterized by the most distinguishable volatile profile among the investigated monovarietal EVOOs. It contained the lowest concentrations of the majority of LOX volatiles (Table 1), including the most potent odorants with the highest OAVs (Table 2), which was certainly a direct cause of the lowest intensities of the majority of positive odor attributes perceived in this EVOO (Figure 3, Table S3). On the other hand, it was found to have high amounts of hexanal, particular C6 alcohols, and hexyl acetate, compounds often accounted among the carriers of green odor which derive from the enzymatic degradation of linoleic acid but also oxidation $[10,28,55]$. Lastovka EVOO contained the highest concentrations of particular monoterpenes and sesquiterpenes, whose sensory contribution is generally described by descriptors such as citrus, camphor, eucalyptus, roses, etc., as well as wood. Although sensory relevance of terpenes in olive oil is currently still unknown and it is certainly limited by the lipid matrix in which these lipophilic molecules are highly soluble, the possibility of their contribution to the specific wood odor perceived in Lastovka EVOO during sensory analysis (Figure 3, Table S3) should not be excluded. Several terpenes were extracted by PLSDA as among the most discriminative compounds for this variety (Figure 2). Particular sesquiterpenes, on the other hand, such as $(+)$-cycloisosativene, $\alpha$-copaene, $\alpha$-muurolene, $\delta$-cadinene, and several unidentified ones were found in the lowest concentrations in Lastovka EVOO (Table 1). Terpenes were previously found to have large potential to differentiate EVOO according to variety [16,56], which was basically confirmed in this study. Other compounds found to be characteristic for Lastovka EVOO, such as particular saturated short-chain aldehydes, ketones, alcohols, and acids, could also have had a sensory impact with their malty, pungent, rancid, and sweaty nuances (Table 2).

One of probably the most important characteristics found typical for Leccino EVOO was the ratio between the important (E)-2- and (Z)-3-C6 forms, which was generally the highest and discriminated well this EVOO from the majority of the other studied EVOOs (Tables 1 and 2). The highest concentration of (E)-2-hexenal and the lowest concentration of (Z)-3-hexenal, (Z)-3-hexen-1-ol, as well as the low concentration of (Z)-3-hexenyl acetate in Leccino EVOO were likely the result of high (Z)-3:(E)-2-enal isomerase activity in Leccino olives, i.e., olive paste during milling and malaxation steps [57]. Considering that the estimated contribution of (E)-2-hexenal to the aroma of EVOOs was generally lower than that of (Z)-3-hexenal (Table 2), it is possible that one of the consequences of the observed differences was a slightly lower intensity of particular odor sensory attributes, such as green/grass leaves and chicory/rocket, observed in Leccino with respect to the EVOOs from the other, native Istrian varieties Buža, I. bjelica and Rosinjola (Figure 3, Table S3). Other interesting features of Leccino EVOO included lower concentrations of particular sesquiterpenes and furanoids. In fact, many sesquiterpenes were among those with the highest VIP scores extracted by PLSDA, but with a negative sign (Figure 2).

Phenols, especially secoiridoids, are responsible for the characteristic EVOO bitterness and pungency, but the specific sensory contribution of each individual major secoiridoid has not been precisely elucidated up to date. Nevertheless, there is solid evidence that $p$-HPEA-EDA is a key contributor to pungency, while the pungency of other, monoaldehydic ligstroside aglycons is weaker, although still strong [24]. Ligstroside aglycons were found to generally be less bitter than pungent, which was especially the case for $p$-HPEA-EDA. In the same study [24] it was found that the majority of 
oleuropein aglycons, including 3,4-DHPEA-EDA, was described as both bitter and pungent, with some of them exhibiting rather strong bitterness. The lowest intensity of bitterness observed in Oblica and Leccino (Figure 2, Table S3) could be tentatively linked to the lowest oleuropein aglycon I concentrations found in these EVOO (Table 3). The intensity of pungency did not quite correlate with the average p-HPEA-EDA concentrations (Figure 3, Table 3, Table S3). In contrast to I. bjelica, for which a positive correlation was observed, Rosinjola EVOO was characterized as intensively pungent according to the official method [1] (intensity $>6$ ) despite containing relatively low concentration of this secoiridoid. The highest average concentration of oleuropein aglycon II in Rosinjola EVOO (Table 3) could have possibly compensated for this deficiency. The pungency of I. bjelica possibly partly originated also from the highest concentrations of all the three monoaldehydic ligstroside aglycons found in this EVOO (Table 3). Especially interesting was the highest ratio of bitterness to pungency found in Lastovka EVOO (Figure 3, Table S3). Roughly, Lastovka EVOO contained among the highest concentrations of oleuropein aglycones and among the lowest concentrations of ligstroside aglycones, which could have had such an impact. This EVOO had the highest concentration of $p$-coumaric acid which, although relatively low, possibly contributed to the bitterness observed. It is worth mentioning that the UPLC chromatograms of Lastovka EVOO contained several unidentified peaks in addition to those observed in the other monovarietal EVOOs (data not shown), which possibly originated from the compounds with sensory relevance. The so-called sweetness in most cases coincided with the lower amounts of total phenols, which was as expected (Figure 3, Table 3). Again, several features turned out to be specific for Leccino EVOO (Table 3), the most important being the highest concentration of 3,4-DHPEA-EDA, which implied variety-dependent differences with respect to the availability of precursors and enzymatic activity between Leccino and Croatian native olive varieties.

\section{Conclusions}

The use of GC-IT-MS and UPLC-DAD proved to be a powerful combination for studying the inter-varietal diversity of typical volatile and phenolic profiles of Croatian EVOOs, respectively. Each of the investigated monovarietal EVOO displayed unique volatile aroma and phenol composition. The qualitative and quantitative chromatographic data was useful for tentative elucidation of some of the perceived sensory attributes including the variety-typical ones, which though has to be taken with caution due to the extreme complexity of the established chemical profiles with the majority of volatiles with still unknown sensory relevance. Many potential varietal markers were extracted by uniand multivariate statistical analysis despite high intra-varietal heterogeneity. It was demonstrated that volatiles and phenols from all the investigated chemical classes can be useful for this purpose. Many of the volatile compounds which turned out to have a notable discrimination power were (tentatively) identified for the first time in EVOO, or were generally neglected in previous studies, especially from sensorial point of view. In fact, only in a few cases were the major LOX compounds, studied most extensively among the volatiles up to date, sufficient for a robust varietal differentiation in this work. This indicates a large potential of the untargeted fingerprinting approach for EVOO characterization, differentiation, and authentication studies.

The number of the extracted robust varietal markers among the investigated chemical compounds largely exceeded the number of typical sensory attributes useful to differentiate monovarietal EVOOs. It is reasonable to conclude that the approach which comprises GC-IT-MS and UPLC-DAD analytical techniques may provide additional objective information about varietal origin which successfully complement those obtained by sensory analysis. Probably the best example for this is the case of Rosinjola EVOO which was relatively similar and hardly distinguishable from that of Buža variety based solely on the sensory analysis, but was characterized by many exclusive chemical markers among benzenoid and furanoid volatiles which discriminated this EVOO rather successfully.

The results obtained in this study could certainly be useful for improving the quality management and control in the production of Croatian monovarietal/PDO EVOO. These findings could contribute 
to strengthening their PDO identities and position on the market, and could be especially useful for discriminating EVOOs of Croatian native varieties from the world famous Leccino variety.

Supplementary Materials: The following are available online at http://www.mdpi.com/2304-8158/8/11/565/s1, Table S1: Climate parameters in the Istria and Dalmatia regions of Croatia in 2015, Table S2: Standardized coefficients of the variables selected for the differentiation of monovarietal Buža, Istarska bjelica, Rosinjola, Oblica, Lastovka, and Leccino extra virgin olive oils on the first three discriminant functions obtained by stepwise linear discriminant analysis, and the percentage of correct classification at each step, Table S3: The intensities and scores of the sensory attributes perceived in monovarietal extra virgin olive oils produced from Buža, Istarska bjelica, Rosinjola, Oblica, Lastovka, and Leccino varieties in Croatia.

Author Contributions: Conceptualization, I.L.; Methodology, I.L.; Formal analysis, I.L., M.L., M.Ž., M.K., S.G., K.B.B.; Resources, I.L., M.L., K.B.B.; Data curation, I.L., M.L., K.B.B.; Writing-original draft preparation, I.L.; Writing-review and editing, I.L., M.L., K.B.B.; Supervision, I.L.; Project administration, I.L.; Funding acquisition, I.L.

Funding: This research was funded by Croatian Science Foundation grant number UIP-2014-09-1194.

Acknowledgments: The authors would like to thank olive oil producers from Istria and Dalmatia (Croatia, EU) for donating the samples of extra virgin olive oils, and Ivana Horvat M.Sc. for technical assistance.

Conflicts of Interest: The authors declare no conflict of interest. The funders had no role in the design of the study; in the collection, analyses, or interpretation of data; in the writing of the manuscript, or in the decision to publish the results.

\section{References}

1. European Economic Community. Commission Regulation (EEC) No 2568/91 of 11 July 1991 (and later modifications) on the characteristics of olive oil and olive-residue oil and the relevant methods of analysis. Off. J. Eur. Communities 1991, L248, 1-83.

2. European Union. Regulation (EU) No 1151/2012 of the European parliament and of the Council of 21 November 2012 (and later modifications) on quality schemes for agricultural products and foodstuffs. Off. J. Eur. Union 2012, L343, 1-29.

3. International Olive Council. COI/T.20/Doc. No. 22 Method for the Organoleptic Assessment of Extra Virgin Olive Oil Applying to Use a Designation of Origin; International Olive Oil Council: Madrid, Spain, 2005.

4. Daisa, P.; Hatzakis, E. Quality assessment and authentication of virgin olive oil by NMR spectroscopy: A critical review. Anal. Chim. Acta 2013, 765, 1-27. [CrossRef] [PubMed]

5. Aparicio, R.; Morales, M.T.; Aparicio-Ruiz, R.; Tena, N.; García-González, D.L. Authenticity of olive oil: Mapping and comparing official methods and promising alternatives. Food Res. Int. 2013, 54, 2025-2038. [CrossRef]

6. López-Cortés, I.; Salazar-García, D.C.; Velázquez-Martí, B.; Salazar, D.M. Chemical characterization of traditional varietal olive oils in East of Spain. Food Res. Int. 2013, 54, 1934-1940. [CrossRef]

7. Olmo-García, L.; Polari, J.J.; Li, X.; Bajoub, A.; Fernández-Gutiérrez, A.; Wang, S.C.; Carrasco-Pancorbo, A. Study of the minor fraction of virgin olive oil by a multi-class GC-MS approach: Comprehensive quantitative characterization and varietal discrimination potential. Food Res. Int. 2019, 125, 108649. [CrossRef]

8. Kritioti, A.; Menexes, G.; Drouza, C. Chemometric characterization of virgin olive oils of the two major Cypriot cultivars based on their fatty acid composition. Food Res. Int. 2018, 103, 426-437. [CrossRef]

9. Angerosa, F. Virgin olive oil odour notes: Their relationships with volatile compounds from the lipoxygenase pathway and secoiridoid compounds. Food Chem. 2000, 68, 283-287. [CrossRef]

10. Angerosa, F. Influence of volatile compounds on virgin olive oil quality evaluated by analytical approaches and sensor panels. Eur. J. Lipid Sci. Technol. 2002, 104, 639-660. [CrossRef]

11. Angerosa, F.; Campestre, C. Sensory quality: Methodologies and applications. In Handbook of Olive Oil-Analysis and Properties; Aparicio, R., Harwood, J., Eds.; Springer: New York, NY, USA, 2013; pp. 523-560.

12. Campestre, C.; Angelini, G.; Gasbarri, C.; Angerosa, F. The compounds responsible for the sensory profile in monovarietal virgin olive oils. Molecules 2017, 22, 1833. [CrossRef]

13. Servili, M.; Montedoro, G. Contribution of phenolic compounds to virgin olive oil quality. Eur. J. Lipid Sci. Technol. 2002, 104, 602-613. [CrossRef]

14. Taticchi, A.; Esposto, S.; Servili, M. The basis of the sensory properties of virgin olive oil. In Olive Oil Sensory Science; Monteleone, E., Langstaff, S., Eds.; John Wiley \& Sons, Ltd.: Hoboken, NJ, USA, 2014; pp. 33-54. 
15. Blasi, F.; Pollini, L.; Cossignani, L. Varietal authentication of extra virgin olive oils by triacylglycerols and volatiles analysis. Foods 2019, 8, 58. [CrossRef] [PubMed]

16. Cecchi, T.; Alfei, B. Volatile profiles of Italian monovarietal extra virgin olive oils via HS-SPME-GC-MS: Newly identified compounds, flavors molecular markers, and terpenic profile. Food Chem. 2013, 141, 2025-2035. [CrossRef] [PubMed]

17. García-González, D.L.; Romero, N.; Aparicio, R. Comparative study of virgin olive oil quality from single varieties cultivated in Chile and Spain. J. Agric. Food Chem. 2010, 58, 12899-12905. [CrossRef] [PubMed]

18. Ocakoglu, D.; Tokatli, F.; Ozen, B.; Korel, F. Distribution of simple phenols, phenolic acids and flavonoids in Turkish monovarietal extra virgin olive oils for two harvest years. Food Chem. 2009, 113, 401-410. [CrossRef]

19. Piscopo, A.; De Bruno, A.; Zappia, A.; Ventre, C.; Poiana, M. Characterization of monovarietal olive oils obtained from mills of Calabria region (Southern Italy). Food Chem. 2016, 213, 313-318. [CrossRef] [PubMed]

20. Sagratini, G.; Maggi, F.; Caprioli, G.; Cristalli, G.; Ricciutelli, M.; Torregiani, E.; Vittori, S. Comparative study of aroma profile and phenolic content of Montepulciano monovarietal red wines from the Marches and Abruzzo regions of Italy using HS-SPME-GC-MS and HPLC-MS. Food Chem. 2012, 132, 1592-1599. [CrossRef] [PubMed]

21. Angerosa, F.; Servili, M.; Selvaggini, R.; Taticchi, A.; Esposto, S.; Montedoro, G. Volatile compounds in virgin olive oil: Occurrence and their relationship with the quality. J. Chromatogr. A 2004, 1054, 17-31. [CrossRef]

22. Kalua, C.M.; Allen, M.S.; Bedgood, D.R.; Bishop, A.G.; Prenzler, P.D.; Robards, K. Olive oil volatile compounds, flavour development and quality: A critical review. Food Chem. 2007, 100, 273-286. [CrossRef]

23. Vichi, S.; Pizzale, L.; Conte, L.S.; Buxaderas, S.; López-Tamames, E. Solid-Phase microextraction in the analysis of virgin olive oil volatile fraction: Characterization of virgin olive oils from two distinct geographical areas of Northern Italy. J. Agric. Food Chem. 2003, 51, 6572-6577. [CrossRef]

24. Andrewes, P.; Busch, J.L.H.C.; De Joode, T.; Groenewegen, A.; Alexandre, H. Sensory properties of virgin olive oil polyphenols: Identification of deacetoxy-ligstroside aglycon as a key contributor to pungency. J. Agric. Food Chem. 2003, 51, 1415-1420. [CrossRef] [PubMed]

25. Bendini, A.; Cerretani, L.; Carrasco-Pancorbo, A.; Gómez-Caravaca, A.M.; Segura-Carretero, A.; Fernández-Gutiérrez, A.; Lercker, G. Phenolic molecules in virgin olive oils: A survey of their sensory properties, health effects, antioxidant activity and analytical methods. An overview of the last decade. Molecules 2007, 12, 1679-1719. [CrossRef] [PubMed]

26. Amirante, P.; Clodoveo, M.L.; Tamborrino, A.; Leone, A.; Dugo, G. Oxygen concentration control during olive oil extraction process: A new system to emphasize the organoleptic and healthy properties of virgin olive oil. Acta Hortic. 2012, 949, 473-480. [CrossRef]

27. Taticchi, A.; Esposto, S.; Veneziani, G.; Urbani, S.; Selvaggini, R.; Servili, M. The influence of the malaxation temperature on the activity of polyphenoloxidase and peroxidase and on the phenolic composition of virgin olive oil. Food Chem. 2013, 136, 975-983. [CrossRef] [PubMed]

28. Angerosa, F.; Basti, C.; Vito, R. Virgin olive oil volatile compounds from lipoxygenase pathway and characterization of some Italian cultivars. J. Agric. Food Chem. 1999, 47, 836-839. [CrossRef] [PubMed]

29. Cirilli, M.; Caruso, G.; Gennai, C.; Urbani, S.; Frioni, E.; Ruzzi, M.; Servili, M.; Gucci, R.; Poerio, E.; Muleo, R. The role of polyphenoloxidase, peroxidase, and $\beta$-glucosidase in phenolics accumulation in Olea europaea $\mathrm{L}$. fruits under different water regimes. Front. Plant Sci. 2017, 8, 717. [CrossRef] [PubMed]

30. Ministry of Agriculture, Republic of Croatia. Available online: https://poljoprivreda.gov.hr/istaknute-teme/ hrana-111/oznake-kvalitete/zoi-zozp-zts-poljoprivrednih-i-prehrambenih-proizvoda/zasticena-oznakaizvornosti-zoi/1206 (accessed on 9 October 2019).

31. International Olive Council, Madrid, Spain. Available online: http://www.internationaloliveoil.org/estaticos/ view/131-world-olive-oil-figures (accessed on 9 October 2019).

32. Poljuha, D.; Sladonja, B.; Šetić, E.; Milotić, A.; Bandelj, D.; Jakše, J.; Javornik, B. DNA fingerprinting of olive varieties in Istria (Croatia) by microsatellite markers. Sci. Hortic. 2008, 115, 223-230. [CrossRef]

33. Brkić Bubola, K.; Koprivnjak, O.; Sladonja, B.; Lukić, I. Volatile compounds and sensory profiles of monovarietal virgin olive oils from Buža, Črna and Rosinjola cultivars in Istria (Croatia). Food Technol. Biotechnol. 2012, 50, 192-198.

34. Giacometti, J.; Milin, Č.; Giacometti, F.; Ciganj, Z. Characterisation of monovarietal olive oils obtained from Croatian cvs. Drobnica and Buža during the ripening period. Foods 2018, 7, 188. [CrossRef] 
35. Kulišić Bilušić, T.; Melliou, E.; Giacometti, J.; Čaušević, A.; Čorbo, S.; Landeka, M.; Magiatis, P. Phenolics, fatty acids, and biological potential of selected Croatian EVOOs. Eur. J. Lipid Sci. Technol. 2017, 119, 1700108. [CrossRef]

36. Lukić, I.; Carlin, S.; Horvat, I.; Vrhovsek, U. Combined targeted and untargeted profiling of volatile aroma compounds with comprehensive two-dimensional gas chromatography for differentiation of virgin olive oils according to variety and geographical origin. Food Chem. 2019, 270, 403-414. [CrossRef] [PubMed]

37. Lukić, I.; Krapac, M.; Lukić, M.; Vrhovsek, U.; Godena, S.; Brkić Bubola, K. Towards understanding the varietal typicity of virgin olive oil by correlating sensory and compositional analysis data: A case study. Food Res. Int. 2018, 112, 78-89. [CrossRef] [PubMed]

38. Šarolić, M.; Gugić, M.; Friganović, E.; Tuberoso, C.I.G.; Jerković, I. Phytochemicals and other characteristics of Croatian monovarietal extra virgin olive oils from Oblica, Lastovka and Levantinka varieties. Molecules 2015, 20, 4395-4409. [CrossRef] [PubMed]

39. Žanetić, M.; Cerretani, L.; Škevin, D.; Politeo, O.; Vitanović, E.; Jukić Špika, M.; Perica, S.; Ožić, M. Influence of polyphenolic compounds on the oxidative stability of virgin olive oils from selected autochthonous varieties. J. Food Agric. Environ. 2013, 11, 126-131.

40. Žanetić, M.; Škevin, D.; Vitanović, E.; Jukić Špika, M.; Perica, S. Survey of phenolic compounds and sensorial profile of Dalmatian virgin olive oils. Pomol. Croat. 2011, 17, 19-30.

41. Brkić Bubola, K.; Koprivnjak, O.; Sladonja, B.; Škevin, D.; Belobrajić, I. Chemical and sensorial changes of Croatian monovarietal olive oils during ripening. Eur. J. Lipid Sci. Technol. 2012, 114, 1400-1408. [CrossRef]

42. Jerman Klen, T.; Golc Wondra, A.; Vrhovšek, U.; Mozetič Vodopivec, B. Phenolic profiling of olives and olive oil process-derived matrices using UPLC-DAD-ESI-QTOF-HRMS analysis. J. Agric. Food Chem. 2015, 63, 3859-3872. [CrossRef]

43. Xia, J.; Sinelnikov, I.; Han, B.; Wishart, D.S. MetaboAnalyst 3.0-Making metabolomics more meaningful. Nucleic Acids Res. 2015, 43, W251-W257. [CrossRef]

44. Aparicio, R.; Luna, G. Characterization of monovarietal virgin olive oils. Eur. J. Lipid Sci. Technol. 2002, 104, 614-627. [CrossRef]

45. Morales, M.T.; Luna, G.; Aparicio, R. Comparative study of virgin olive oil sensory defects. Food Chem. 2005, 91, 293-301. [CrossRef]

46. Reiners, J.; Grosch, W. Odorants of virgin olive oils with different flavor profiles. J. Agric. Food Chem. 1998, 46, 2754-2763. [CrossRef]

47. Reboredo-Rodríguez, P.; González-Barreiro, C.; Cancho-Grande, B.; Simal-Gándara, J. Improvements in the malaxation process to enhance the aroma quality of extra virgin olive oils. Food Chem. 2014, 158, 534-545. [CrossRef] [PubMed]

48. Inglese, P.; Famiani, F.; Galvano, F.; Servili, M.; Esposto, S.; Urbani, S. Factors affecting extra-virgin olive oil composition. In Horticultural Reviews; Janick, J., Ed.; John Wiley \& Sons Inc.: Hoboken, NJ, USA, 2010; pp. 83-147.

49. Romero, N.; Saavedra, J.; Tapia, F.; Sepúlveda, B.; Aparicio, R. Influence of agroclimatic parameters on phenolic and volatile compounds of Chilean virgin olive oils and characterization based on geographical origin, cultivar and ripening stage. J. Sci. Food Agric. 2016, 96, 583-592. [CrossRef] [PubMed]

50. Beck, J.J.; Higbee, B.S.; Light, D.M.; Gee, W.S.; Merrill, G.B.; Hayashi, J.M. Hull split and damaged almond volatiles attract male and female navel orangeworm moths. J. Agric. Food Chem. 2012, 60, 8090-8096. [CrossRef] [PubMed]

51. Lukić, I.; Krapac, M.; Horvat, I.; Godena, S.; Kosić, U.; Brkić Bubola, K. Three-factor approach for balancing the concentrations of phenols and volatiles in virgin olive oil from a late-ripening olive cultivar. LWT Food Sci. Technol. 2018, 87, 194-202. [CrossRef]

52. Majetić Germek, V.; Koprivnjak, O.; Butinar, B.; Pizzale, L.; Bučar-Miklavčič, M.; Conte, L.S. Influence of phenols mass fraction in olive (Olea europaea L.) paste on volatile compounds in Buža cultivar virgin olive oil. J. Agric. Food Chem. 2013, 61, 5921-5927.

53. Angerosa, F.; Mostallino, R.; Basti, C.; Vito, R. Influence of malaxation temperature and time on the quality of virgin olive oils. Food Chem. 2001, 72, 19-28. [CrossRef]

54. Aparicio, R.; Morales, M.T. Characterization of olive ripeness by green aroma compounds of virgin olive oil. J. Agric. Food Chem. 1998, 46, 1116-1122. [CrossRef] 
55. Hongsoongnern, P.; Chambers, E. A lexicon for green odor or flavor and characteristics of chemicals associated with green. J. Sens. Stud. 2008, 23, 205-221. [CrossRef]

56. Vichi, S.; Guadayol, J.M.; Caixach, J.; Lopez-Tamames, E.; Buxaderas, S. Monoterpene and sesquiterpene hydrocarbons of virgin olive oil by headspace solidphase microextraction coupled to gas chromatography/mass spectrometry. J. Chromatogr. A 2006, 1125, 117-123. [CrossRef]

57. Williams, M.; Salas, J.J.; Sanchez, J.; Harwood, J.L. Lipoxygenase pathway in olive callus cultures (Olea europaea). Phytochemistry 2000, 53, 13-19. [CrossRef]

(C) 2019 by the authors. Licensee MDPI, Basel, Switzerland. This article is an open access article distributed under the terms and conditions of the Creative Commons Attribution (CC BY) license (http://creativecommons.org/licenses/by/4.0/). 